\title{
Phosphate minerals from the hydrothermal quartz veins in specialized S-type granites, Gemerská Poloma (Western Carpathians, Slovakia)
}

\author{
Martin ŠTEVKO ${ }^{*}$, Pavel UHER ${ }^{1}$, Jiří SEJKORA², Radana MALÍKOVÁ2 ${ }^{2,3}$, Radek ŠKODA ${ }^{3}$, \\ Tomáš VACULOVIČ 4
}

\author{
${ }^{1}$ Department of Mineralogy and Petrology, Faculty of Natural Sciences, Comenius University, Ilkovičova 6, 84215 Bratislava, Slovak \\ Republic;stevko@fns.uniba.sk \\ ${ }^{2}$ Department of Mineralogy and Petrology, National Museum, Cirkusová 1740, 19300 Praha 9-Horní Počernice, Czech Republic \\ ${ }^{3}$ Department of Geological Sciences, Faculty of Science, Masaryk University, Kotlářská 2, 61137 Brno, Czech Republic \\ ${ }^{4}$ Department of Chemistry, Faculty of Science, Masaryk University, Kamenice 5, 62500 Brno, Czech Republic \\ * Corresponding author
}

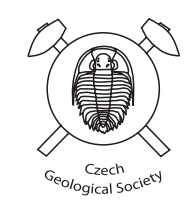

An interesting association of phosphate minerals (fluorapatite, triplite, arrojadite-group minerals and viitaniemiite) was studied from intra-granitic hydrothermal quartz veins with minor amounts of albite, orthoclase, muscovite, fluorite, rhodochrosite, arsenopyrite, pyrite, bismuthinite and kobellite. The veins occur in highly evolved, Permian topaz-zinnwaldite leucogranite at the Elisabeth adit near Gemerská Poloma, Gemeric Unit, Western Carpathians (eastern Slovakia). Fluorapatite is enriched in $\mathrm{Mn}(\sim 4 \mathrm{wt} . \% \mathrm{MnO}, 0.3 \mathrm{apfu} \mathrm{Mn})$ and frequently replaced by triplite, representing the first known triplite occurrence in Western Carpathians. This mineral forms irregular aggregates ( $\leq 7 \mathrm{~cm}$ across), $\mathrm{Mn} /(\mathrm{Mn}+\mathrm{Fe}$ ) atomic ratio of which attains 0.68 to 0.78 and $\mathrm{F} /(\mathrm{F}+\mathrm{OH})=0.89-0.92$. "Fluorarrojadite-(BaNa)" to its Mn-dominant analogue "fluordickinsonite-(BaNa)" (both minerals still not approved by IMACNMNC) occurs as aggregates up to $2 \mathrm{~cm}$ across, showing Sr-rich $(\sim 1.7 \mathrm{wt}$. \% SrO, $\sim 0.36$ apfu $\mathrm{Sr})$ and Sr-poor $(\leq 0.6$ wt. $\% \mathrm{SrO}, \leq 0.13$ apfu $\mathrm{Sr})$ compositions with $W$ site $\mathrm{F} /(\mathrm{F}+\mathrm{OH})=0.74-0.80$ and $M$ site $\mathrm{Mn} /(\mathrm{Mn}+\mathrm{Fe})=0.39-0.52$. Rare viitaniemiite is Mn-rich (10 to $11 \mathrm{wt}$. \% MnO, 0.34-0.38 apfu Mn). The phosphate mineralization in quartz represents a high-temperature hydrothermal assemblage. The F-rich $\mathrm{Mn}, \mathrm{Fe}, \mathrm{Ca}$-bearing phosphates, fluorite, and muscovite precipitated most likely in presence of alkali- and fluorine-bearing post-magmatic fluids which altered primary magmatic minerals (especially Li-rich micas and alkali feldspars) and liberated some elements ( $\mathrm{Fe}, \mathrm{Mn}, \mathrm{Al}$, $\mathrm{Ba}, \mathrm{Sr}, \mathrm{Na}, \mathrm{K}$ ) from the adjacent granite.

Keywords: hydrothermal phosphates, triplite, arrojadite group, chemical composition, S-type granite, Slovak Republic Received: 24 July, 2015; accepted: 9 December, 2015; handling editor: I. Broska

\section{Introduction}

Phosphate minerals represent widespread minor to accessory constituents of various magmatic to hydrothermal systems, especially in highly fractionated, P-rich peraluminous granite-pegmatite suites and greisenized granites, commonly with orogenic and post-orogenic S-type affinity (e.g., London 1997; Breiter et al. 1997, 2005; Piccoli and Candela 2002; Sejkora et al. 2006; Petrík et al. 2011; Baijot et al. 2012; Llorens and Moro 2012; Roda-Robles et al. 2012). Textural relationships, internal zoning and compositional variations of principal phosphate minerals (mainly apatite-group minerals, amblygonite, triphylite-lithiophyllite, triplite-zwieselite, and other Fe-Mn phosphates) represent sensitive indicators of magmatic to hydrothermal evolution in such systems. Specific $\mathrm{P}-\mathrm{T}-\mathrm{X}-f \mathrm{O}_{2}$ conditions lead to precipitation of unique chemical compositions of some phosphate minerals, for example in the arrojadite-group minerals (Chopin et al.
2006). Therefore, detailed knowledge of the nature and chemistry of phosphate minerals is an important tool for our understanding of magmatic to hydrothermal processes and role of phosphorus in highly evolved silicic rocks (London 1992, 1997; Breiter et al. 1997, 2005).

In the present work, we describe phosphate mineral association (fluorapatite, triplite, arrojadite-group minerals and viitaniemiite) from intra-granitic hydrothermal quartz veins in the highly fractionated topaz-zinnwaldite leucogranites at the Elisabeth adit near Gemerská Poloma, eastern Slovakia. The paper documents presence of rare phosphate minerals: "fluorarrojadite-(BaNa)" together with its Mn-dominant analogue "fluordicskinsonite(BaNa)" (both are phases not yet approved by IMACNMNC) and viitaniemiite. Similar minerals have been recently described in the nearby Surovec Li-F granite by Petrík et al. (2011). Still, the phosphate mineralization at Gemerská Poloma represents the first reported occurrence of triplite in the Western Carpathians. 


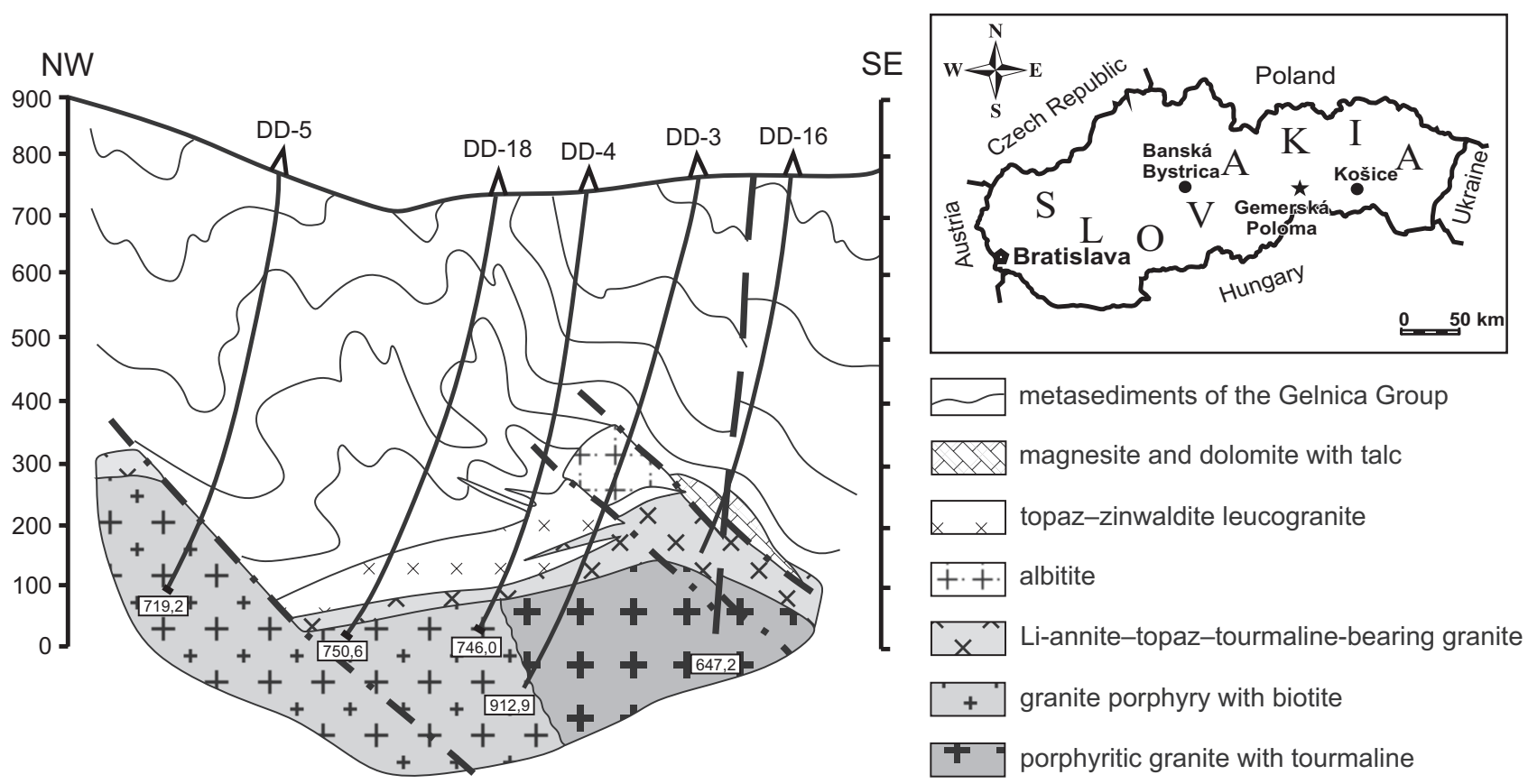

Fig. 1 Location and NW-SE cross section of the granite pluton near Gemerská Poloma (modified after Dianiška et al. 2002).

\section{Geological setting}

The hydrothermal quartz veins are developed in the specialized Gemeric granites, which were recently located during the driving of Elisabeth adit at the Gemerská Poloma talc deposit, located about $10 \mathrm{~km}$ north-west of Rožňava, Gemeric Unit, Slovak Republic [GPS $48^{\circ} 45^{\prime} 4.07^{\prime \prime} \mathrm{N}$ and $\left.20^{\circ} 29^{\prime} 39.32^{\prime \prime} \mathrm{E}\right]$.

The granitic rocks of the Gemeric Unit represent a distinct type of specialized ( $\mathrm{Sn}-\mathrm{W}-\mathrm{F})$, highly evolved suite with S-type affinity that differs from other granitoids occurring in the Veporic and Tatric Units of the Western Carpathian crystalline basement. Besides fluorine, they are enriched in phosphorus and rare lithophile elements, such as $\mathrm{Li}, \mathrm{Rb}, \mathrm{Cs}, \mathrm{B}, \mathrm{Ga}, \mathrm{Sn}, \mathrm{W}, \mathrm{Nb}, \mathrm{Ta}, \mathrm{U}$ and depleted in REE, Zr, Ti, Sr, Ba (e.g., Uher and Broska 1996; Petrík and Kohút 1997; Kubiš and Broska 2005, 2010). Recent zircon $\mathrm{U}-\mathrm{Pb}$ and molybdenite $\mathrm{Re}-\mathrm{Os}$ isotopic dating indicate emplacement of the Gemeric granites and related post-magmatic mineralization during Late Permian ( 260 to $230 \mathrm{Ma}$; Poller et al. 2002; Kohút and Stein 2005; Gaab et al. 2006). Younger, Alpine (Cretaceous) fluiddriven low-temperature tectono-metamorphic overprint affected the granitic rocks along mylonite zones (Breiter et al. 2015).

The Gemeric granitic rocks form several small plutons that intruded the intensively folded Lower Paleozoic (mainly Ordovician to Devonian) volcano-sedimentary complex of the Gelnica Group, metamorphosed under greenschist-facies metamorphic conditions (Bajaník et al. 1984; Petrasová et al. 2007). In the investigated Gemerská Poloma area, the metamorphic rocks are composed mainly of phyllites, metapyroclastic rocks of rhyolitic to dacitic composition, locally with lenses of metadolomite and strongly steatitized magnesite. The latter have been recently exploited as a talc deposit near Gemerská Poloma (Kilík 1997). Several types of granites were distinguished in the studied area (Fig. 1): (1) coarse-grained porphyritic granite to granite porphyry, (2) medium-grained Li-annite-topaz-tourmaline bearing granite, (3) P-enriched topaz-zinnwaldite leucogranite and (4) albitite (Dianiška et al. 2002, 2007; Petrík et al. 2014; Breiter et al. 2015).

Except for albitites, all listed granitoid types were found in the Elisabeth adit. Hydrothermal quartz veins with albite, muscovite, fluorite, carbonates, sulfides and sulfosalts were observed in all types of granite, but the occurrence of quartz veins with phosphate mineralization is limited only to the topaz-zinnwaldite leucogranite. The veins with phosphates are up to $8 \mathrm{~cm}$ thick and more than $3 \mathrm{~m}$ long and in addition to phosphates they also contain minor amounts of albite, orthoclase, muscovite, fluorite, carbonates (Mn-rich siderite, rhodochrosite and dolomite), arsenopyrite, pyrite, bismuthinite and kobellite.

Tab. 1 Refined unit-cell parameters of triplite from Gemerská Poloma in comparison with published data

\begin{tabular}{lrrr}
\hline & this work & Waldrop (1969) & Downs (2006) \\
\hline$a[\AA]$ & $12.085(7)$ & $12.065(1)$ & $12.036(2)$ \\
$b[\AA]$ & $6.463(5)$ & $6.454(1)$ & $6.4646(3)$ \\
$c[\AA]$ & $9.941(6)$ & $9.937(1)$ & $9.965(1)$ \\
$\beta\left[^{\circ}\right]$ & $107.09(4)$ & $107.093(6)$ & $106.75(1)$ \\
$V\left[\AA^{3}\right]$ & $742.1(6)$ & 739.59 & $742.5(1)$ \\
\hline
\end{tabular}

* Fe and Mg-rich triplite 

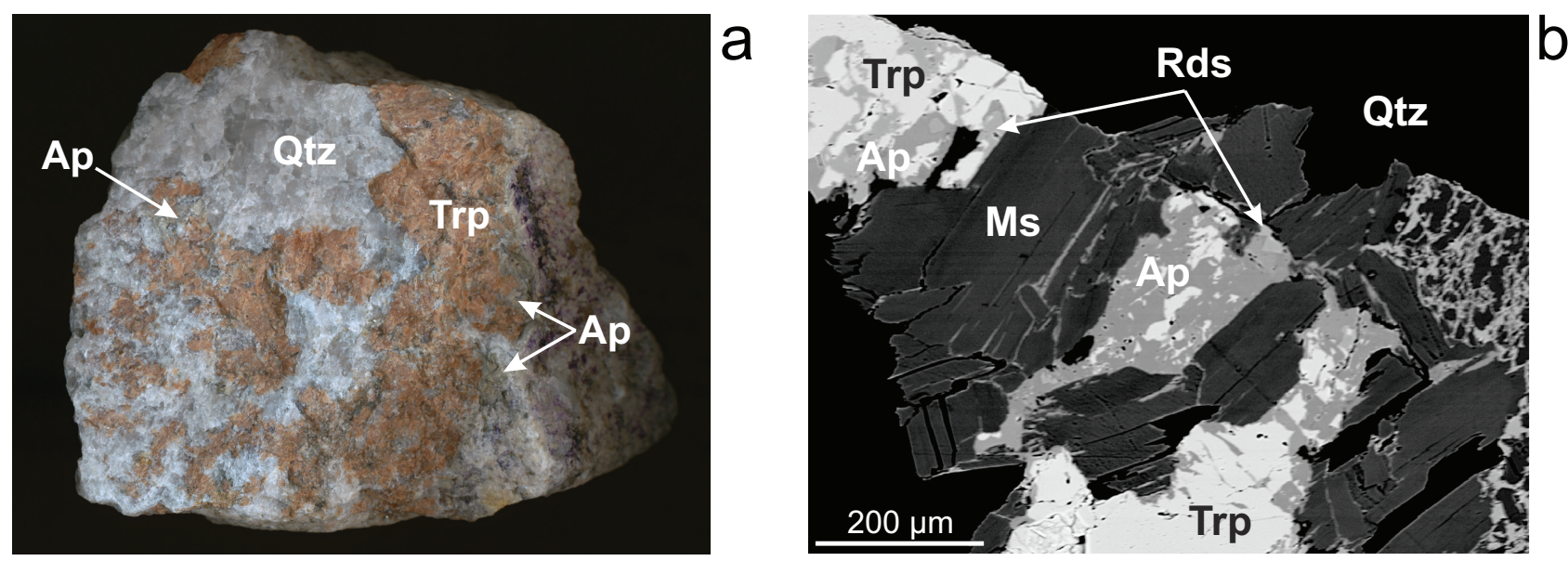

Fig. 2 Triplite from Gemerská Poloma. a - Brown-red subhedral to anhedral grains and aggregates of triplite (Trp) replacing green-grey fluorapatite (Ap) enclosed in quartz (Qtz), width of image: $20 \mathrm{~cm}$; b - Homogenous aggregates of triplite (Trp) replacing fluorapatite (Ap) associated with muscovite (Ms) and minor rhodochrosite (Rds), BSE image.

\section{Analytical methods}

The morphology of minerals was studied with the Olympus SZ-61 optical microscope in combination with the Olympus SP-350 digital camera (Department of Mineralogy and Petrology, Faculty of Natural Sciences, Comenius University in Bratislava, Slovakia), used for photography in incandescent light.

The powder X-ray diffraction (P-XRD) data for phosphates were obtained on a Bruker D8 Advance diffractometer equipped with solidstate LynxEye detector and secondary monochromator producing $\mathrm{Cu} K_{\alpha}$ radiation housed at the same institution. The instrument was operating at 40 $\mathrm{kV}$ and $40 \mathrm{~mA}$. In order to minimize the background, the powder samples were placed on the surface of a flat silicon wafer in ethanol suspension. Positions and intensities of diffractions were processed using a pseudo-Voigt shape function with the High-Score Plus (PANalytical) program. Unit-cell parameters were refined by leastsquare method with the Celref program (Laugier and Bochu 2011). For the arrojadite group minerals, unit-cell parameters were refined using structural data published by Chopin et al. (2006) for fluorarrojadite-(BaFe).

Quantitative chemical analyses of phosphates were carried out using a Cameca SX100 electron microprobe (Laboratory of Electron Microscopy and Microanalysis of the Masaryk
University and Czech Geological Survey in Brno, Czech Republic) in the wavelength-dispersion mode. Operating conditions were $15 \mathrm{kV}, 10-20 \mathrm{nA}$ and a 5-10 $\mu \mathrm{m}$ beam diameter. The following lines and standards were used: fluorapatite ( $\mathrm{P}, \mathrm{Ca} K_{\alpha}$ ), GaAs (As $L_{\alpha}$ ), spessartine ( $\left.\mathrm{Si}, \mathrm{Mn} K_{\alpha}\right)$, titanite (Ti $\left.K_{\alpha}\right)$, sanidine (Al, K $\left.K_{\alpha}\right)$, gahnite (Zn $K_{\alpha}$ ), almandine $\left(\mathrm{Fe} K_{\alpha}\right), \mathrm{Mg}_{2} \mathrm{SiO}_{4}\left(\mathrm{Mg} \mathrm{K}_{\alpha}\right), \mathrm{SrSO}_{4}$ $\left(\mathrm{Sr} \mathrm{L}_{\alpha}\right)^{2}$, barite $(\mathrm{Ba} L \beta)$, albite $\left(\mathrm{Na} K_{\alpha}\right)$, topaz $\left(\mathrm{F} K_{\alpha}\right)$, and

Tab. 2 Chemical composition of triplite from Gemerská Poloma (in wt. \%)

\begin{tabular}{|c|c|c|c|c|c|c|}
\hline Anal. \# & 117 & 118 & 119 & 129 & 130 & 131 \\
\hline $\mathrm{P}_{2} \mathrm{O}_{5}$ & 32.30 & 32.42 & 32.83 & 32.83 & 32.61 & 32.75 \\
\hline $\mathrm{FeO}$ & 19.71 & 19.41 & 17.62 & 13.70 & 13.62 & 14.80 \\
\hline $\mathrm{MnO}$ & 42.30 & 40.74 & 44.58 & 48.39 & 49.01 & 47.03 \\
\hline $\mathrm{MgO}$ & 0.46 & 0.44 & 0.44 & 0.34 & 0.33 & 0.32 \\
\hline $\mathrm{CaO}$ & 1.40 & 1.64 & 1.08 & 1.04 & 0.99 & 1.11 \\
\hline $\mathrm{H}_{2} \mathrm{O}$ calc. & 0.38 & 0.44 & 0.35 & 0.38 & 0.36 & 0.41 \\
\hline $\mathrm{F}$ & 7.82 & 7.72 & 8.04 & 7.86 & 7.97 & 7.91 \\
\hline $\mathrm{Cl}$ & 0.03 & 0.06 & 0.00 & 0.00 & 0.00 & 0.00 \\
\hline $\mathrm{O}=\mathrm{F}$ & -3.29 & -3.25 & -3.39 & -3.31 & -3.36 & -3.33 \\
\hline $\mathrm{O}=\mathrm{Cl}$ & -0.01 & -0.01 & 0.00 & 0.00 & 0.00 & 0.00 \\
\hline Total & 101.10 & 99.61 & 101.55 & 100.78 & 101.53 & 101.00 \\
\hline $\mathrm{P}^{5+}$ & 1.000 & 1.000 & 1.000 & 1.000 & 1.000 & 1.000 \\
\hline $\mathrm{Fe}^{2+}$ & 0.603 & 0.591 & 0.530 & 0.418 & 0.413 & 0.446 \\
\hline $\mathrm{Mn}^{2+}$ & 1.310 & 1.257 & 1.359 & 1.495 & 1.504 & 1.437 \\
\hline $\mathrm{Mg}^{2+}$ & 0.025 & 0.024 & 0.024 & 0.018 & 0.018 & 0.017 \\
\hline $\mathrm{Ca}^{2+}$ & 0.055 & 0.064 & 0.042 & 0.041 & 0.038 & 0.043 \\
\hline $\operatorname{Sum} A$ & 1.993 & 1.936 & 1.955 & 1.972 & 1.973 & 1.943 \\
\hline $\mathrm{OH}^{-}$ & 0.094 & 0.107 & 0.085 & 0.093 & 0.087 & 0.098 \\
\hline $\mathrm{F}^{-}$ & 0.904 & 0.890 & 0.915 & 0.907 & 0.913 & 0.902 \\
\hline $\mathrm{Cl}^{-}$ & 0.002 & 0.004 & 0.000 & 0.000 & 0.000 & 0.000 \\
\hline Sum $X$ & 1.000 & 1.001 & 1.000 & 1.000 & 1.000 & 1.000 \\
\hline $\mathrm{O}^{2-}$ & 3.993 & 3.936 & 3.954 & 3.972 & 3.972 & 3.943 \\
\hline Sum cat. & 2.993 & 2.936 & 2.955 & 2.972 & 2.973 & 2.943 \\
\hline Sum an. & 4.993 & 4.937 & 4.954 & 4.972 & 4.972 & 4.943 \\
\hline $\mathrm{Mn} /(\mathrm{Mn}+\mathrm{Fe})$ & 0.68 & 0.68 & 0.72 & 0.78 & 0.78 & 0.76 \\
\hline
\end{tabular}

Formulae based on $\mathrm{P}=1$ apfu and $(\mathrm{OH}+\mathrm{F}+\mathrm{Cl})=1$ apfu 

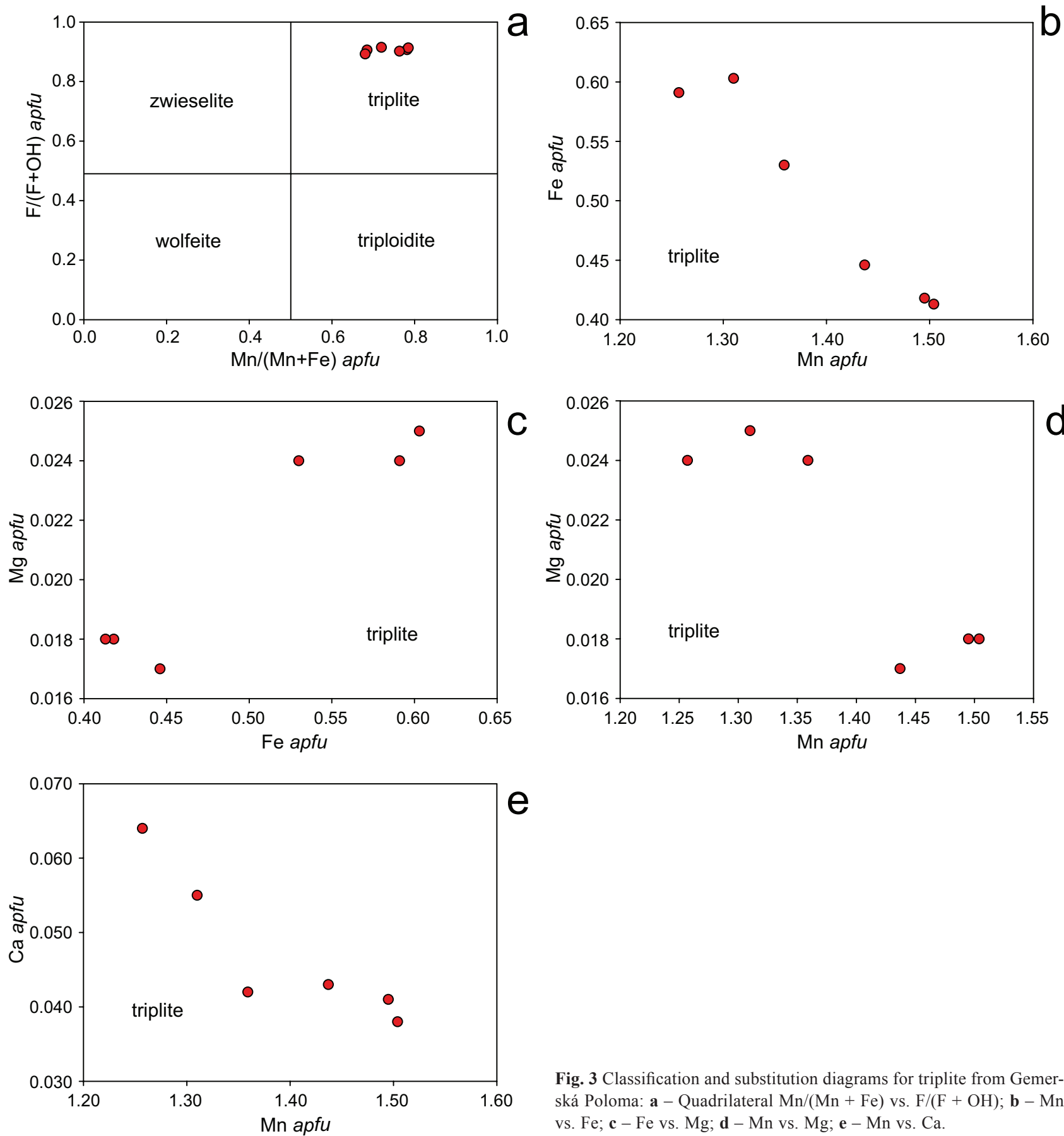

Fig. 3 Classification and substitution diagrams for triplite from Gemerská Poloma: a - Quadrilateral $\mathrm{Mn} /(\mathrm{Mn}+\mathrm{Fe})$ vs. $\mathrm{F} /(\mathrm{F}+\mathrm{OH}) ; \mathbf{b}-\mathrm{Mn}$ vs. $\mathrm{Fe} ; \mathbf{c}-\mathrm{Fe}$ vs. $\mathrm{Mg}$; d $-\mathrm{Mn}$ vs. $\mathrm{Mg} ; \mathbf{e}-\mathrm{Mn}$ vs. $\mathrm{Ca}$.

vanadinite $\left(\mathrm{Cl} K_{\alpha}\right)$. Raw intensities were converted to the concentrations using automatic PAP matrix correction (Pouchou and Pichoir 1985).

Major- and trace-element concentrations in phosphate samples were determined by LA-ICP-MS at the Department of Chemistry, Faculty of Science, Masaryk University, Brno (Czech Republic). The setup consists of laser-ablation system UP213 (New Wave, USA) and quadrupole ICP-MS Agilent 7500ce (Agilent Technologies, Japan). Ablation system is equipped with $\mathrm{Nd}: \mathrm{YAG}$

laser emitting radiation with wavelength of $213 \mathrm{~nm}$. The sample is placed in ablation cell (Supercell ${ }^{\circledR}$, New Wave, USA) where the interaction of laser radiation with sample occurs. The ablated material is transported by carrier gas (helium) with flow rate of $1.0 \mathrm{l} / \mathrm{min}$ into ICP-MS. The sample gas (argon) with flow rate of $0.6 \mathrm{l} / \mathrm{min}$ is admixed to the carrier gas flow after ablation cell. The ICP-MS conditions (gas flow rates, sampling depth and electrostatic lenses voltages of the mass spectrometer) were optimized in order to maximize signal/noise $(\mathrm{S} / \mathrm{N})$ 
ratio and to achieve counts ratios of $\mathrm{ThO}^{+} / \mathrm{Th}^{+}$and $\mathrm{U}^{+} / \mathrm{Th}^{+}$lower than 0.2 and $1.1 \%$, respectively. All LAICP-MS measurements were done in single hole drilling mode with laser spot diameter of $40 \mu \mathrm{m}$, laser fluence of $10 \mathrm{~J} / \mathrm{cm}^{2}$ and repetition rate of 10 $\mathrm{Hz}$. For quantification purpose the certified reference material NIST SRM 612 was used. All measurements were normalized to average Ca concentration in triplite (8 638 ppm) and arrojadite-group minerals $(14,598 \mathrm{ppm})$ measured by the electron microprobe.

\section{Results}

\subsection{Fluorapatite}

Fluorapatite is the most common phosphate in quartz veins. It forms green-grey, subhedral to anhedral grains (Fig. 2a) and aggregates up to $1.5 \mathrm{~cm}$ in size, which are enclosed in quartz, and frequently replaced by triplite (Fig. 2b). Rare are well developed, bluish-green prismatic fluorapatite crystals up to $2 \mathrm{~mm}$ in drusy cavities. Fluorapatite is homogenous in back-scattered electrons (BSE) mode (Fig. 2b); it shows $\sim 1$ apfu F, distinct enrichment in $\mathrm{Mn}$ (around 0.3 apfu, 3.8 to 4.3 wt. $\% \mathrm{MnO}$ ) and low Fe content $(\sim 0.03$ apfu, 0.3 to 0.5 wt. $\% \mathrm{FeO})$.

\subsection{Triplite}

This mineral forms salmon-pink to red-brown subhedral to anhedral grains, up to $3.5 \mathrm{~cm}$ (Fig. $2 \mathrm{a}$ ) or irregular aggregates up to $7 \mathrm{~cm}$, which are embedded in massive quartz together with fluorapatite, minerals of arrojadite group, Fe-rich rhodochrosite, muscovite, arsenopyrite and $\mathrm{Bi}$ sulfosalts. Triplite is homogenous in BSE and it often replaces older aggregates of fluorapatite (Fig. 2b).

Triplite was confirmed by the P-XRD and its refined unit-cell parameters (Tab. 1) agree well with the published data and confirm the (1b) structure observed
Tab. 3 LA-ICP-MS concentrations of elements in triplite (Trp) and arrojadite-group minerals (Arr) from Gemerská Poloma (in ppm)

\begin{tabular}{|c|c|c|c|c|c|c|c|c|c|c|}
\hline Mineral & $\operatorname{Trp}$ & $\operatorname{Trp}$ & Trp & Trp & Trp & Trp & Trp & Trp & Trp & $\begin{array}{l}\text { det. } \\
\text { lim. }\end{array}$ \\
\hline Anal. \# & 1 & 2 & 3 & 4 & 5 & 6 & 7 & 8 & 9 & \\
\hline$\overline{\mathrm{Li}}$ & 8 & 7 & 7 & 4 & 7 & 9 & 8 & 13 & 13 & 2.9 \\
\hline $\mathrm{Be}$ & $<$ & $<$ & $<$ & $<$ & $<$ & $<$ & $<$ & $<$ & $<$ & 1.7 \\
\hline B & $<$ & $<$ & $<$ & $<$ & $<$ & $<$ & $<$ & $<$ & $<$ & 15 \\
\hline $\mathrm{Na}$ & $<$ & $<$ & $<$ & $<$ & 155 & $<$ & $<$ & 158 & $<$ & 145 \\
\hline $\mathrm{Mg}$ & 2766 & 2723 & 1489 & 1455 & 2195 & 1719 & 2119 & 2330 & 2429 & 1.1 \\
\hline $\mathrm{Al}$ & 25 & 3 & 12 & 14 & 4 & 4 & $<$ & 8 & 3 & 1.6 \\
\hline $\mathrm{Si}$ & 426 & $<$ & $<$ & $<$ & $<$ & $<$ & $<$ & $<$ & $<$ & 280 \\
\hline $\mathrm{K}$ & $<$ & $<$ & $<$ & $<$ & $<$ & $<$ & $<$ & $<$ & $<$ & 59 \\
\hline $\mathrm{Sc}$ & 145 & 132 & 109 & 115 & 116 & 294 & 218 & 246 & 181 & 1 \\
\hline $\mathrm{Ti}$ & 190 & 189 & 100 & 145 & 173 & 499 & 321 & 428 & 255 & 3 \\
\hline $\mathrm{V}$ & $<$ & $<$ & $<$ & $<$ & $<$ & $<$ & $<$ & $<$ & $<$ & 0.1 \\
\hline $\mathrm{Cr}$ & $<$ & $<$ & $<$ & $<$ & $<$ & $<$ & $<$ & $<$ & $<$ & 1.4 \\
\hline $\mathrm{Co}$ & $<$ & $<$ & $<$ & $<$ & $<$ & $<$ & $<$ & $<$ & $<$ & 0.1 \\
\hline $\mathrm{Ni}$ & $<$ & $<$ & $<$ & $<$ & $<$ & $<$ & $<$ & $<$ & $<$ & 2.6 \\
\hline $\mathrm{Cu}$ & $<$ & $<$ & $<$ & $<$ & $<$ & $<$ & $<$ & $<$ & $<$ & 0.8 \\
\hline $\mathrm{Zn}$ & 359 & 330 & 236 & 231 & 309 & 379 & 314 & 470 & 356 & 0.1 \\
\hline $\mathrm{Ga}$ & $<$ & $<$ & $<$ & $<$ & $<$ & $<$ & $<$ & $<$ & $<$ & 0.1 \\
\hline $\mathrm{Ge}$ & $<$ & $<$ & $<$ & $<$ & $<$ & $<$ & $<$ & $<$ & $<$ & 2.9 \\
\hline As & $<$ & $<$ & $<$ & $<$ & $<$ & $<$ & $<$ & $<$ & $<$ & 2.9 \\
\hline $\mathrm{Rb}$ & 1.7 & 1.4 & 1.5 & 2.0 & 2.2 & $<$ & $<$ & 1.5 & 1.6 & 1.1 \\
\hline $\mathrm{Sr}$ & 4.8 & 1.8 & 37 & 47 & 1.4 & 0.7 & 0.9 & 1.0 & 1.0 & 0.1 \\
\hline $\mathrm{Y}$ & 3.2 & 2.8 & 2.0 & 1.9 & 2.1 & 1.4 & 2.6 & 2.8 & 2.6 & 0.1 \\
\hline $\mathrm{Zr}$ & 0.1 & $<$ & $<$ & $<$ & 0.2 & 0.3 & $<$ & 0.6 & $<$ & 0.1 \\
\hline $\mathrm{Nb}$ & 5.1 & 3.6 & 3.5 & 7.1 & 3.7 & 18.4 & 8.9 & 24 & 9.8 & 0.1 \\
\hline $\mathrm{Sn}$ & 17 & 14 & 9.2 & 6.5 & 8.9 & 6.4 & 8.3 & 13 & 14 & 2.9 \\
\hline $\mathrm{Sb}$ & 1.2 & 0.5 & 0.6 & 0.7 & 0.7 & 0.4 & 0.1 & 1.0 & 0.5 & 0.1 \\
\hline $\mathrm{Ba}$ & 2.2 & 2.0 & 5.6 & 10 & 1.7 & 1.1 & 0.4 & $<$ & 0.3 & 0.1 \\
\hline $\mathrm{La}$ & $<$ & $<$ & $<$ & $<$ & $<$ & $<$ & $<$ & $<$ & $<$ & 0.1 \\
\hline $\mathrm{Ce}$ & $<$ & $<$ & $<$ & $<$ & $<$ & $<$ & $<$ & $<$ & $<$ & 0.1 \\
\hline $\operatorname{Pr}$ & $<$ & $<$ & $<$ & $<$ & $<$ & $<$ & $<$ & $<$ & $<$ & 0.1 \\
\hline $\mathrm{Nd}$ & $<$ & $<$ & $<$ & $<$ & $<$ & $<$ & $<$ & $<$ & $<$ & 0.1 \\
\hline $\mathrm{Sm}$ & $<$ & 0.2 & 0.1 & $<$ & $<$ & 0.2 & $<$ & 0.4 & $<$ & 0.1 \\
\hline $\mathrm{Eu}$ & $<$ & $<$ & $<$ & $<$ & $<$ & $<$ & $<$ & $<$ & 0.5 & 0.1 \\
\hline $\mathrm{Gd}$ & 0.3 & 0.4 & 0.2 & 0.3 & $<$ & $<$ & $<$ & 0.3 & $<$ & 0.1 \\
\hline $\mathrm{Tb}$ & 0.1 & $<$ & $<$ & $<$ & $<$ & $<$ & 0.1 & $<$ & $<$ & 0.1 \\
\hline Dy & 0.5 & 0.1 & 0.3 & 0.1 & 0.2 & 0.2 & $<$ & 0.4 & 0.4 & 0.1 \\
\hline Но & $<$ & $<$ & $<$ & $<$ & $<$ & $<$ & $<$ & $<$ & $<$ & 0.1 \\
\hline $\mathrm{Er}$ & 0.2 & 0.3 & 0.2 & 0.2 & 0.1 & $<$ & 0.2 & 0.3 & $<$ & 0.1 \\
\hline $\mathrm{Tm}$ & $<$ & $<$ & $<$ & $<$ & $<$ & $<$ & $<$ & $<$ & $<$ & 0.1 \\
\hline $\mathrm{Yb}$ & 0.5 & 0.3 & 0.1 & 0.4 & $<$ & 0.3 & 0.5 & 0.7 & 0.8 & 0.1 \\
\hline $\mathrm{Lu}$ & $<$ & 0.1 & $<$ & $<$ & $<$ & $<$ & $<$ & $<$ & $<$ & 0.1 \\
\hline Hf & $<$ & $<$ & $<$ & $<$ & $<$ & $<$ & $<$ & $<$ & $<$ & 0.1 \\
\hline $\mathrm{Ta}$ & 0.2 & 0.1 & 0.2 & 0.4 & $<$ & 3.0 & 1.4 & 5.5 & 0.9 & 0.1 \\
\hline $\mathrm{Pb}$ & $<$ & $<$ & 0.8 & 0.6 & 0.6 & 0.6 & $<$ & $<$ & $<$ & 0.1 \\
\hline Th & 0.2 & 0.1 & $<$ & 0.1 & 0.1 & $<$ & 0.2 & 0.1 & $<$ & 0.1 \\
\hline $\mathrm{U}$ & 2.1 & 0.8 & 2.7 & 5.4 & 0.3 & 1.3 & 0.4 & 3.5 & 1.0 & 0.1 \\
\hline
\end{tabular}

$<=$ below detection limit (det. lim.)
0.1

0.1

9

.9

0.1

. 1

0.1

1

0.1

1

0.1

.1

0.1

for F-rich poor-Mg members of the wagnerite group (Lazic et al 2014). According to electron-microprobe measurements, triplite $\mathrm{Mn} /(\mathrm{Mn}+\mathrm{Fe})$ atomic ratio attains $0.68-0.78$ and $\mathrm{F} /(\mathrm{F}+\mathrm{OH})$ ratio $0.89-0.92$ (Tab. 2; Fig. 3a). Concentrations of $\mathrm{Mg}$ are negligible (around 
Tab. 3 LA-ICP-MS concentrations of elements in triplite (Trp) and arrojadite-group minerals (Arr) from Gemerská Poloma (in ppm) (continued)

\begin{tabular}{|c|c|c|c|c|c|c|c|c|c|c|}
\hline Mineral & Arr & Arr & Arr & Arr & Arr & Arr & Arr & Arr & Arr & Arr \\
\hline Anal. \# & 1 & 2 & 3 & 4 & 5 & 6 & 7 & 8 & 9 & 10 \\
\hline $\mathrm{Li}$ & 1330 & 1394 & 1248 & 1281 & 1318 & 1426 & 1290 & 1112 & 1330 & 1117 \\
\hline $\mathrm{Be}$ & 3.9 & 3.9 & 3.7 & 4.4 & 2.3 & 3.1 & 3.7 & 3.2 & 3.9 & 3.8 \\
\hline B & 16 & $<$ & $<$ & $<$ & $<$ & $<$ & $<$ & $<$ & $<$ & $<$ \\
\hline $\mathrm{Na}$ & 33826 & 34843 & 32288 & 34512 & 34558 & 32079 & 31463 & 35304 & 36088 & 30321 \\
\hline $\mathrm{Mg}$ & 539 & 542 & 515 & 590 & 504 & 589 & 567 & 583 & 555 & 545 \\
\hline $\mathrm{Al}$ & 10333 & 9817 & 9912 & 10725 & 9826 & 10766 & 11013 & 10934 & 10749 & 10177 \\
\hline $\mathrm{Si}$ & 360 & 501 & $<$ & $<$ & $<$ & $<$ & $<$ & $<$ & $<$ & $<$ \\
\hline $\mathrm{K}$ & 7158 & 7200 & 6937 & 7174 & 7200 & 7028 & 6409 & 6637 & 7612 & 6783 \\
\hline $\mathrm{Sc}$ & 1028 & 973 & 885 & 1023 & 955 & 1167 & 973 & 1068 & 1028 & 979 \\
\hline $\mathrm{Ti}$ & 188 & 196 & 177 & 196 & 196 & 132 & 164 & 163 & 219 & 181 \\
\hline $\mathrm{V}$ & $<$ & $<$ & $<$ & $<$ & $<$ & $<$ & $<$ & $<$ & $<$ & $<$ \\
\hline $\mathrm{Cr}$ & $<$ & $<$ & $<$ & $<$ & $<$ & $<$ & $<$ & $<$ & $<$ & $<$ \\
\hline $\mathrm{Co}$ & 0.1 & $<$ & 0.2 & 0.2 & 0.1 & 0.2 & 0.2 & 3.0 & 0.3 & 0.4 \\
\hline $\mathrm{Ni}$ & $<$ & $<$ & $<$ & $<$ & $<$ & $<$ & $<$ & $<$ & $<$ & $<$ \\
\hline $\mathrm{Cu}$ & 1.5 & 1.0 & 1.7 & 1.3 & 1.0 & 2.3 & 1.4 & 3.1 & 1.6 & 1.4 \\
\hline $\mathrm{Zn}$ & 1813 & 1967 & 1861 & 1960 & 1792 & 1907 & 1790 & 1981 & 1685 & 1737 \\
\hline $\mathrm{Ga}$ & 343 & 326 & 330 & 367 & 329 & 431 & 421 & 450 & 361 & 335 \\
\hline $\mathrm{Ge}$ & $<$ & $<$ & $<$ & $<$ & $<$ & $<$ & $<$ & $<$ & $<$ & $<$ \\
\hline As & $<$ & 2.9 & $<$ & $<$ & 3.0 & $<$ & $<$ & $<$ & $<$ & $<$ \\
\hline $\mathrm{Rb}$ & 77 & 98 & 70 & 87 & 81 & 69 & 68 & 73 & 81 & 84 \\
\hline $\mathrm{Sr}$ & 10141 & 9763 & 10216 & 11337 & 10942 & 9371 & 8898 & 9304 & 9159 & 10725 \\
\hline $\mathrm{Y}$ & 20 & 21 & 19 & 25 & 20 & 19 & 17 & 19 & 28 & 25 \\
\hline $\mathrm{Zr}$ & 0.8 & 0.8 & 0.9 & 0.9 & 0.7 & 0.5 & 0.7 & 0.7 & 0.8 & 0.7 \\
\hline $\mathrm{Nb}$ & 0.3 & 0.2 & 0.3 & 0.4 & 0.6 & 0.4 & 0.3 & 0.3 & 0.4 & 0.2 \\
\hline $\mathrm{Sn}$ & 11 & 9.7 & 8.0 & 11 & 7.0 & 7.4 & 8.0 & 8.3 & 11.2 & 8.8 \\
\hline $\mathrm{Sb}$ & 0.2 & $<$ & 0.1 & $<$ & 0.4 & 0.1 & 0.2 & $<$ & 0.3 & 0.2 \\
\hline $\mathrm{Ba}$ & 20686 & 20705 & 21574 & 21384 & 20336 & 25053 & 24797 & 26739 & 22139 & 19478 \\
\hline $\mathrm{La}$ & 2.9 & 2.9 & 2.7 & 3.2 & 2.6 & 2.6 & 2.4 & 2.5 & 3.3 & 3.1 \\
\hline $\mathrm{Ce}$ & 4.6 & 4.7 & 4.3 & 5.2 & 5.1 & 4.3 & 4.5 & 4.1 & 7.2 & 6.4 \\
\hline $\mathrm{Pr}$ & 0.5 & 0.4 & 0.4 & 0.5 & 0.6 & 0.4 & 0.4 & 0.6 & 0.6 & 0.7 \\
\hline $\mathrm{Nd}$ & 1.3 & 1.2 & 1.2 & 1.5 & 1.7 & 1.0 & 1.5 & 1.0 & 2.4 & 2.2 \\
\hline $\mathrm{Sm}$ & 0.5 & 0.8 & 0.7 & 0.5 & 0.8 & 0.4 & 0.7 & 0.6 & 0.7 & 1.2 \\
\hline $\mathrm{Eu}$ & 0.5 & 0.7 & 0.6 & 0.6 & 0.6 & 0.9 & 0.8 & 1.0 & 0.8 & 0.5 \\
\hline $\mathrm{Gd}$ & 0.4 & 0.5 & 0.5 & 0.2 & 0.8 & 0,9 & 0,9 & 0,8 & 1,4 & 0,8 \\
\hline $\mathrm{Tb}$ & 0.2 & 0.3 & 0.3 & 0.3 & 0.2 & 0.4 & 0.2 & 0.3 & 0.3 & 0.3 \\
\hline Dy & 2.1 & 2.4 & 2.0 & 1.7 & 2.6 & 1.9 & 2.0 & 2.3 & 2.9 & 2.9 \\
\hline Ho & 0.6 & 0.6 & 0.4 & 0.6 & 0.5 & 0.4 & 0.5 & 0.5 & 0.7 & 0.5 \\
\hline $\mathrm{Er}$ & 1.9 & 2.3 & 1.8 & 2.6 & 2.7 & 1.5 & 1,9 & 2.2 & 3.0 & 2.7 \\
\hline $\mathrm{Tm}$ & 0.7 & 0.6 & 0.6 & 0.8 & 0.5 & 0.7 & 0,4 & 0.6 & 0.9 & 0.7 \\
\hline $\mathrm{Yb}$ & 6.6 & 9.2 & 7.8 & 10.2 & 8.7 & 7.2 & 7,8 & 6.1 & 9.8 & 9.8 \\
\hline $\mathrm{Lu}$ & 1.3 & 1.5 & 1.2 & 1.5 & 1.3 & 1.3 & 1.2 & 1.3 & 1.4 & 1.6 \\
\hline Hf & $<$ & $<$ & $<$ & $<$ & 0 & $<$ & $<$ & 0 & $<$ & $<$ \\
\hline $\mathrm{Ta}$ & $<$ & $<$ & $<$ & $<$ & 0 & $<$ & $<$ & $<$ & $<$ & $<$ \\
\hline $\mathrm{Pb}$ & 3309 & 3267 & 3127 & 3552 & 3424 & 2694 & 2565 & 2734 & 2984 & 3249 \\
\hline $\mathrm{Th}$ & 0.2 & 0.1 & 0.2 & 0.2 & 0.1 & 0.1 & 0.2 & 0.2 & 0.1 & 0.2 \\
\hline $\mathrm{U}$ & 1.3 & 1.2 & 1.3 & 1.6 & 0.8 & 1.1 & 1.4 & 2.2 & 1.6 & 2.3 \\
\hline
\end{tabular}

substitutions between divalent cations: $\mathrm{FeMn}_{-1}, \mathrm{MgFe}_{-1}, \mathrm{MgMn}_{-1}$ and $\mathrm{CaMn}_{-1}$ (Fig. 3b-e).

As shown by LA-ICP-MS analyses, triplite contains relatively low concentrations of trace elements: $\sim 110$ to 300 ppm Sc, 100 to $500 \mathrm{ppm} \mathrm{Ti}$ and 230 to $470 \mathrm{ppm} \mathrm{Zn;} \mathrm{concentrations} \mathrm{of} \mathrm{other}$ measured trace elements are lower than 20 ppm (Tab. 3).

\section{3. "Fluorarrojadite-(BaNa)" to "fluordickinsonite -(BaNa)"}

Minerals of arrojadite group occur as greenish-yellow to yellowish-brown anhedral grains and aggregates up to $2 \mathrm{~cm}$ in quartz veins (Fig. 4a), associated with fluorapatite, triplite, bismuthinite, kobellite and arsenopyrite. They are only slightly inhomogeneous in BSE (Fig. 4b), where the lighter zones are Ba-enriched. In some cases, they contain irregular microscopic aggregates of viitaniemiite and $\mathrm{Fe}$-rich rhodochrosite. Graphic-like intergrowths of arrojadite group minerals and rhodochrosite were also observed (Fig. 4c).

The P-XRD data (Tab. 4) as well as refined unit-cell parameters of arrojadite-group minerals from the Gemerská Poloma are consistent with those reported from other localities (Tab. 5). Minerals of the arrojadite group have a very complex crystal chemistry and generalised formula of $A_{2} B_{2} \mathrm{CaNa}_{2+\mathrm{x}} M_{13} \mathrm{Al}\left(\mathrm{PO}_{4}\right)_{11}\left(\mathrm{PO}_{3} \mathrm{OH}\right) W_{2}$, where $A$ ( $A 1$ and $A 2)$ sites are occupied by either large divalent cations like $\mathrm{Ba}^{2+}, \mathrm{Sr}^{2+}, \mathrm{Pb}^{2+}$ and vacancy, or monovalent $\left(\mathrm{K}^{+}, \mathrm{Na}^{+}\right)$cations; $B$ site $(B 1$ and $B 2)$ is occupied by smaller divalent cations like $\mathrm{Fe}^{2+}, \mathrm{Mn}^{2+}, \mathrm{Mg}^{2+}$ and vacancy or by monovalent cation $\left(\mathrm{Na}^{+}\right)$. Octahedrally coordinated $M$ site can be occupied by dominant $\mathrm{Fe}^{2+}$ (arrojadite series), $\mathrm{Mn}^{2+}$ (dick-

0.02 apfu). The mineral contains $58-70 \mathrm{~mol}$. \% triplite, 19-27 mol. \% zwieselite, 6-7 mol. \% triploidite and 2-3 mol. \% wolfeite end-member components; the concentrations of other molecules are under 2 mol. \% (Tab. 2). Compositional variations of triplite indicate homovalent insonite series), or $\mathrm{Mg}^{2+}, \mathrm{Zn}^{2+}$ and $\mathrm{Li}^{+}$, whereas the $W$ site represents monovalent anions: $(\mathrm{OH})^{-}$or $\mathrm{F}^{-}$(Cámara et al. 2006; Chopin et al. 2006; Della Ventura et al. 2014). Based on electron-microprobe study, the studied arrojadite-group minerals belong to "fluorar- 

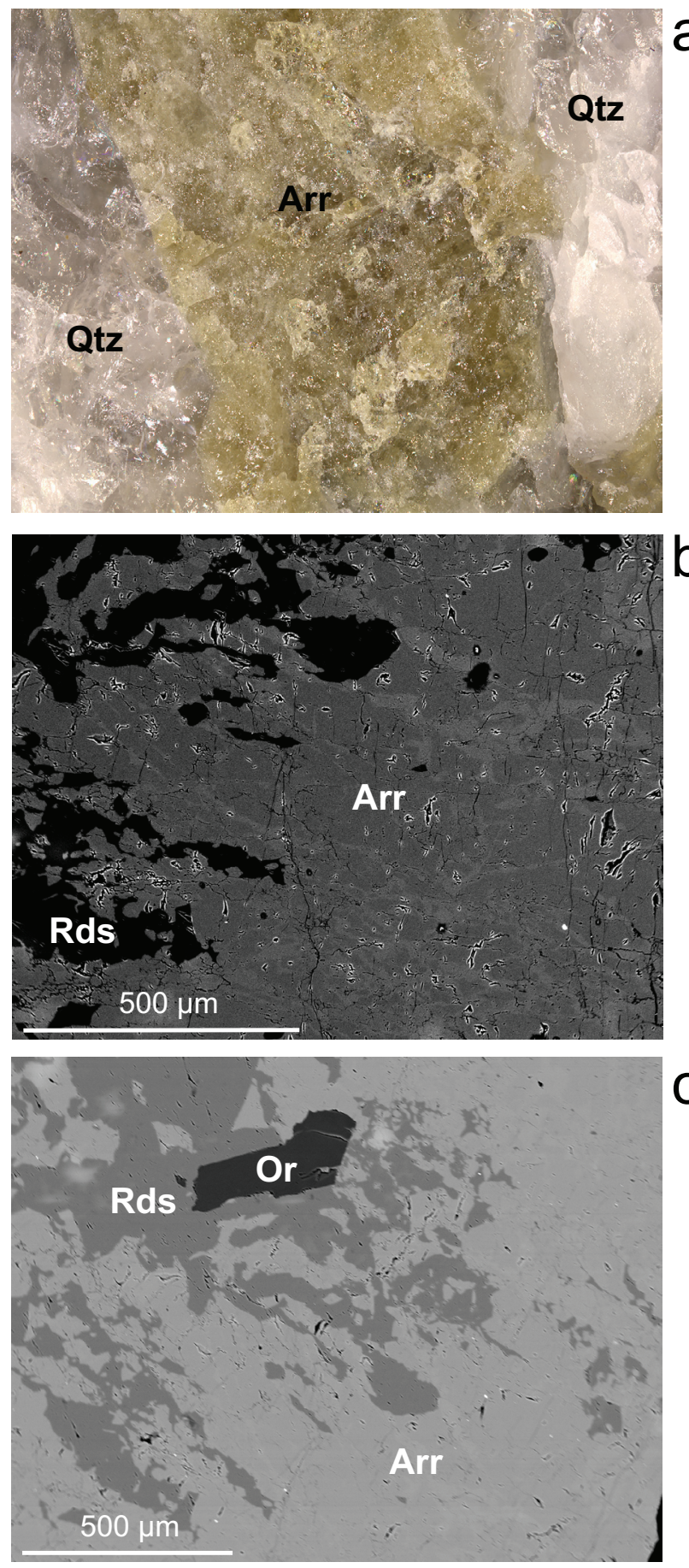

Fig. 4 Arrojadite-group minerals from Gemerská Poloma. a - olivegreen to yellowish-brown aggregate of "fluorarrojadite-(BaNa)" to "fluordickinsonite-(BaNa)" (Arr) enclosed in quartz (Qtz), width of image: $12 \mathrm{~mm}$; b - Slightly irregular chemical zoning of arrojadite-group minerals (Arr) with rhodochrosite (Rds), BSE image; c-Graphic-like intergrowths of arrojadite-group minerals (Arr) with rhodochrosite (Rds) and minor orthoclase (Or), BSE image.

rojadite-(BaNa)" and its Mn-dominant analogue "fluordickinsonite-(BaNa)", These are both IMA-CNMNC unapproved members of arrojadite group, according to the nomenclature of Chopin et al. (2006) (Tab. 6,
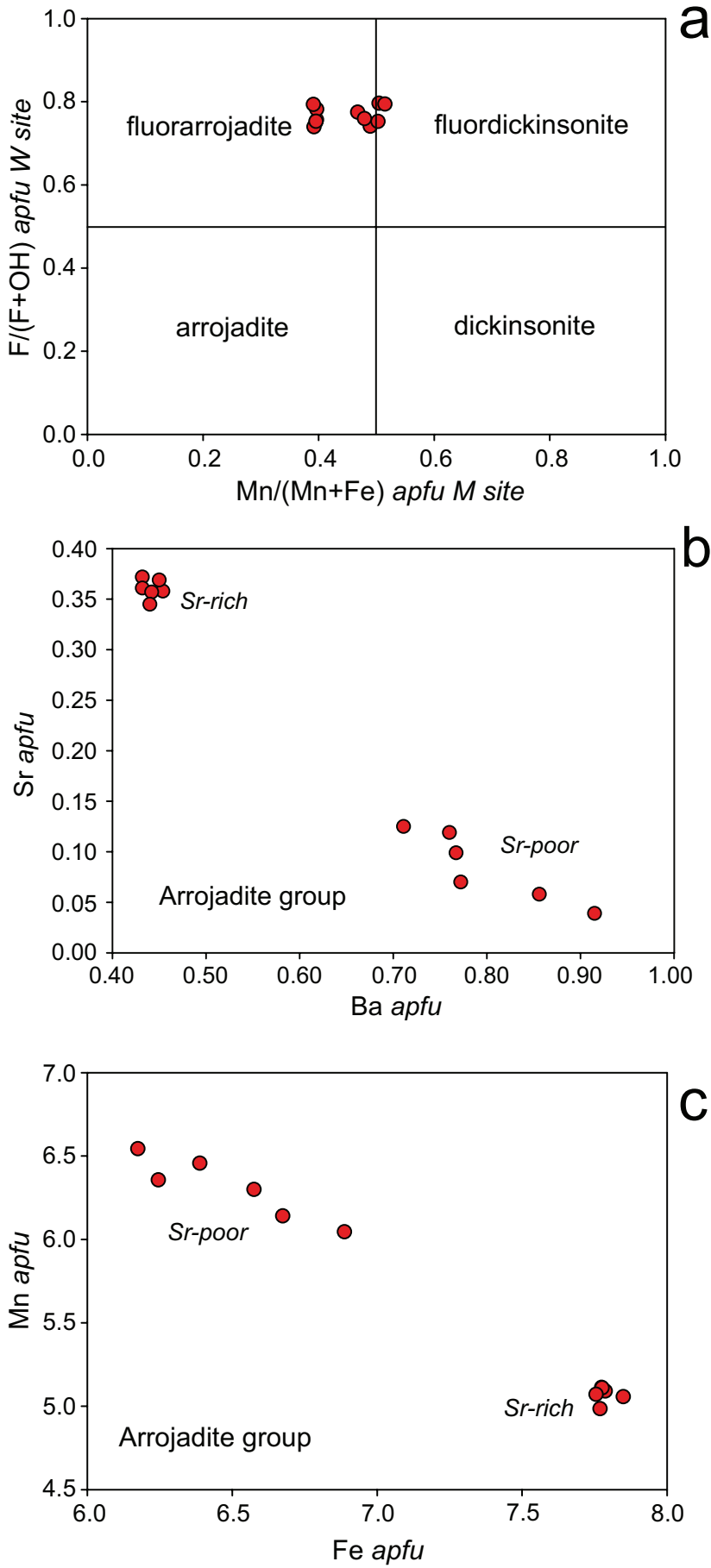

Fig. 5 Classification and substitution diagrams of the arrojadite-group minerals from Gemerská Poloma. a - Quadrilateral $\mathrm{Mn} /(\mathrm{Mn}+\mathrm{Fe})$ vs. $W$ site $\mathrm{F} /(\mathrm{F}+\mathrm{OH}) ; \mathbf{b}-\mathrm{Ba}$ vs. $\mathrm{Sr} ; \mathbf{c}-\mathrm{Fe}$ vs. Mn.

Fig. 5a). Our compositions are fluorine-dominant $[W$ site $\mathrm{F} /(\mathrm{F}+\mathrm{OH})=0.74-0.80]$ and Fe-rich $[\mathrm{Mn} /(\mathrm{Mn}+$ $\mathrm{Fe})=0.39-0.52]$, with high $\mathrm{Ba}$ and $\mathrm{Na}$ contents $(3.0$ to 6.3 wt. $\% \mathrm{BaO}, 0.43-0.92$ apfu $\mathrm{Ba}$ and $\sim 5.5$ wt. \% $\mathrm{Na}_{2} \mathrm{O}, \sim 3.9$ apfu $\left.\mathrm{Na}\right)$. According to $\mathrm{Ba} /(\mathrm{Ba}+\mathrm{Sr})$ atomic ratio, two compositional types could be recognized: Sr-rich "fluorarrojadite-(BaNa)" with $\sim 1.7$ wt.\% $\mathrm{SrO}$ 
Tab. 4 Powder X-ray diffraction pattern of arrojadite-group minerals from Gemerská Poloma

\begin{tabular}{|c|c|c|c|c|c|c|c|c|c|c|c|}
\hline$h$ & $k$ & $l$ & $d_{\mathrm{obs}}$ & $I_{\mathrm{obs}}$ & $d_{\text {calc }}$ & $h$ & $k$ & l & $d_{\mathrm{obs}}$ & $I_{\mathrm{obs}}$ & $d_{\text {calc }}$ \\
\hline$\overline{2}$ & 0 & 2 & 7.586 & 4 & 7.618 & $\overline{5}$ & 1 & 9 & 2.3030 & 1 & 2.3031 \\
\hline$\overline{1}$ & 1 & 2 & 7.361 & 2 & 7.419 & $\overline{5}$ & 3 & 5 & 2.2549 & 5 & 2.2510 \\
\hline 2 & 0 & 2 & 5.908 & 8 & 5.905 & $\overline{3}$ & 3 & 8 & 2.2107 & 2 & 2.2103 \\
\hline$\overline{2}$ & 0 & 4 & 5.487 & 7 & 5.519 & $\overline{7}$ & 1 & 6 & 2.1961 & 9 & 2.1929 \\
\hline$\overline{1}$ & 1 & 4 & 5.211 & 5 & 5.225 & 0 & 2 & 10 & 2.1459 & 18 & 2.1441 \\
\hline 0 & 2 & 0 & 4.997 & 5 & 5.010 & $\overline{4}$ & 4 & 3 & 2.1240 & 6 & 2.1243 \\
\hline$\overline{3}$ & 1 & 2 & 4.749 & 6 & 4.760 & 4 & 4 & 0 & 2.1201 & 7 & 2.1186 \\
\hline 3 & 1 & 0 & 4.683 & 7 & 4.682 & $\overline{4}$ & 4 & 4 & 2.0923 & 1 & 2.0928 \\
\hline 0 & 2 & 2 & 4.604 & 21 & 4.615 & $\overline{2}$ & 0 & 12 & 2.0533 & 2 & 2.0525 \\
\hline 1 & 1 & 4 & 4.554 & 18 & 4.561 & 3 & 3 & 7 & 2.0290 & 2 & 2.0309 \\
\hline 2 & 0 & 4 & 4.235 & 3 & 4.236 & 2 & 4 & 6 & 1.9736 & 6 & 1.9750 \\
\hline$\overline{2}$ & 2 & 2 & 4.181 & 6 & 4.186 & $\overline{2}$ & 4 & 8 & 1.9409 & 3 & 1.9387 \\
\hline 3 & 1 & 2 & 4.046 & 2 & 4.038 & $\overline{7}$ & 3 & 4 & 1.9158 & 8 & 1.9169 \\
\hline 0 & 2 & 4 & 3.824 & 7 & 3.827 & $\overline{3}$ & 1 & 13 & 1.8564 & 10 & 1.8567 \\
\hline 0 & 2 & 5 & 3.445 & 7 & 3.445 & $\overline{1}$ & 3 & 11 & 1.8483 & 5 & 1.8478 \\
\hline 1 & 1 & 6 & 3.402 & 47 & 3.400 & 7 & 1 & 5 & 1.8300 & 1 & 1.8299 \\
\hline$\overline{1}$ & 1 & 7 & 3.320 & 26 & 3.315 & 8 & 2 & 1 & 1.8043 & 1 & 1.8058 \\
\hline 2 & 2 & 4 & 3.240 & 9 & 3.235 & $\overline{5}$ & 1 & 13 & 1.7791 & 2 & 1.7782 \\
\hline 1 & 3 & 1 & 3.217 & 49 & 3.214 & 8 & 2 & 2 & 1.7577 & 23 & 1.7583 \\
\hline$\overline{1}$ & 3 & 2 & 3.197 & 8 & 3.197 & $\overline{4}$ & 2 & 13 & 1.7427 & 4 & 1.7432 \\
\hline 4 & 2 & 0 & 3.115 & 10 & 3.112 & $\overline{6}$ & 2 & 12 & 1.7274 & 5 & 1.7270 \\
\hline$\overline{5}$ & 1 & 4 & 3.033 & 100 & 3.031 & 3 & 3 & 10 & 1.6996 & 3 & 1.7002 \\
\hline 0 & 0 & 8 & 2.965 & 7 & 2.965 & 3 & 1 & 12 & 1.6809 & 3 & 1.6830 \\
\hline$\overline{1}$ & 3 & 4 & 2.932 & 5 & 2.932 & 9 & 1 & 2 & 1.6579 & 10 & 1.6580 \\
\hline 4 & 2 & 2 & 2.873 & 6 & 2.856 & 6 & 4 & 4 & 1.6556 & 8 & 1.6556 \\
\hline$\overline{3}$ & 3 & 2 & 2.846 & 18 & 2.842 & $\overline{2}$ & 6 & 2 & 1.6301 & 2 & 1.6311 \\
\hline$\overline{3}$ & 1 & 8 & 2.826 & 16 & 2.829 & $\overline{3}$ & 1 & 15 & 1.6208 & 1 & 1.6201 \\
\hline$\overline{5}$ & 1 & 6 & 2.772 & 4 & 2.773 & $\overline{5}$ & 5 & 7 & 1.6119 & 6 & 1.6115 \\
\hline 3 & 3 & 1 & 2.757 & 40 & 2.759 & $\overline{6}$ & 4 & 10 & 1.5941 & 2 & 1.5935 \\
\hline 4 & 2 & 3 & 2.709 & 83 & 2.708 & 3 & 5 & 7 & 1.5769 & 3 & 1.5775 \\
\hline 3 & 3 & 2 & 2.669 & 5 & 2.663 & 9 & 1 & 4 & 1.5601 & 5 & 1.5601 \\
\hline 5 & 1 & 3 & 2.613 & 2 & 2.613 & 9 & 3 & 1 & 1.5330 & 3 & 1.5335 \\
\hline$\overline{1}$ & 3 & 6 & 2.592 & 7 & 2.591 & 4 & 6 & 1 & 1.5255 & 2 & 1.5258 \\
\hline 0 & 2 & 8 & 2.553 & 35 & 2.552 & 9 & 3 & 2 & 1.5012 & 2 & 1.5016 \\
\hline 0 & 4 & 0 & 2.507 & 27 & 2.505 & $\overline{3}$ & 5 & 11 & 1.4857 & 4 & 1.4866 \\
\hline$\overline{5}$ & 1 & 8 & 2.4599 & 2 & 2.4583 & $\overline{9}$ & 1 & 13 & 1.4687 & 2 & 1.4685 \\
\hline$\overline{4}$ & 2 & 8 & 2.4167 & 13 & 2.4171 & 7 & 5 & 2 & 1.4580 & 3 & 1.4581 \\
\hline 4 & 2 & 5 & 2.3832 & 7 & 2.3817 & 3 & 3 & 13 & 1.4411 & 3 & 1.4412 \\
\hline$\overline{3}$ & 1 & 10 & 2.3533 & 2 & 2.3546 & $\overline{5}$ & 5 & 11 & 1.4371 & 2 & 1.4368 \\
\hline$\overline{5}$ & 3 & 1 & 2.3349 & 2 & 2.3355 & & & & & & \\
\hline
\end{tabular}

Sr) ratios attain $0.54-0.56$ and $0.85-0.96$, respectively (Tab. 6, Fig. 5b). Potassium shows higher concentration in the Srrich compositions ( 0.9 to 1.0 wt. $\% \mathrm{~K}_{2} \mathrm{O}, 0.40-0.45$ apfu $\left.\mathrm{K}\right)$ in comparison with the Sr-poor analyses ( 0.6 to 0.8 wt. $\% \mathrm{~K}_{2} \mathrm{O}$, $0.29-0.38$ apfu K). Aluminum in the both types attains 0.98 to $1.05 \mathrm{apfu}$, totals of $M$ site cations (Fe, Mn, $\mathrm{Mg}, \mathrm{Zn}, \mathrm{Li}$ ) achieve 13.1 to 13.5. Average lithium content measured by LA-ICP-MS (1285 ppm Li, 0.28 wt. $\% \mathrm{Li}_{2} \mathrm{O}, 0.41 a p f u$ ) is usually lower than theoretical Li contents (0.41-0.62 apfu) calculated according to Chopin et al. (2006), possibly due to uncertainty of measured $\mathrm{Si}$ and $\mathrm{Al}$ contents. Contents of $\mathrm{Ti}, \mathrm{Mg}$, and $\mathrm{Zn}$ are low $(\leq 0.1$ apfu); other measured elements ( $\mathrm{S}, \mathrm{As}, \mathrm{Si}, \mathrm{Cl}$ ) are below the respective detection limits of the electron microprobe. The compositional variations indicate the following substitution mechanisms which also differ between the Sr-rich and Sr-poor compositions: $\mathrm{FeMn}_{-1}$ in $M$ site (Fig. 5c), $\mathrm{BaSr}_{-1}$ in $\mathrm{Al}+\mathrm{Ca}$ sites (Fig. 5b), and $\mathrm{F}(\mathrm{OH})_{-1}$ in $W$ site (Tab. 6).

The LA-ICP-MS analyses of "fluorarrojadite-(BaNa)" to "fluordickinsonite-(BaNa)" reveal elevated concentrations of some elements: $\sim 1100$ to 1400 ppm Li, 2600 to $3600 \mathrm{ppm} \mathrm{Pb}$,

( 0.36 apfu Sr), and Sr-poor "fluorarrojadite-(BaNa)" to "fluordickinsonite-(BaNa)" with 0.2 to $0.6 \mathrm{wt} . \% \mathrm{SrO}$ (0.04 to 0.13 apfu $\mathrm{Sr}$ ). The corresponding $\mathrm{Ba} /(\mathrm{Ba}+$

Tab. 5 Refined unit-cell parameters of arrojadite-group minerals from Gemerská Poloma in comparison with published data

\begin{tabular}{lcc}
\hline & this work & Chopin et al. (2006)* \\
\hline$a[\AA]$ & $16.498(3)$ & $16.4970(9)$ \\
$b[\AA]$ & $10.019(2)$ & $10.0176(5)$ \\
$c[\AA]$ & $24.637(5)$ & $24.6359(13)$ \\
$\beta\left[^{\circ}\right]$ & $105.65(2)$ & $105.649(2)$ \\
$V\left[\AA^{3}\right]$ & $3921(1)$ & $3920.42(5)$ \\
\hline
\end{tabular}

* data for fluorarrojadite-(BaFe)
1700 to $2000 \mathrm{ppm} \mathrm{Zn,} 900$ to $1200 \mathrm{ppm} \mathrm{Sc}, 330$ to $450 \mathrm{ppm} \mathrm{Ga}, 70$ to $100 \mathrm{ppm} \mathrm{Rb}$, and 17 to $28 \mathrm{ppm} \mathrm{Y}$; concentrations of other trace elements are less than 20 ppm (Tab. 3).

\subsection{Viitaniemiite}

Viitaniemiite $\mathrm{Na}(\mathrm{Ca}, \mathrm{Mn}) \mathrm{Al}\left(\mathrm{PO}_{4}\right) \mathrm{F}_{2}(\mathrm{OH})$ is rarest phosphate in the studied association. It occurs as irregular grains and aggregates up to $150 \mu \mathrm{m}$ in size (Fig. 6a), which are enclosed in arrojadite-group minerals together with Fe-rich rhodochrosite. Chemical composition of viitaniemite (Tab. 7) shows a distinct enrichment in 
Tab. 6 Chemical composition of arrojadite-group minerals from Gemerská Poloma (in wt. \%)

\begin{tabular}{|c|c|c|c|c|c|c|c|c|}
\hline Anal. \# & 17 & 18 & 20 & 23 & 30 & 31 & 33 & Average \\
\hline Prefix & Fluor & Fluor & & Fluor & Fluor & Fluor & Fluor & Fluor \\
\hline Root name & arrojadite & arrojadite & arrojadite & arrojadite & arrojadite & dickinsonite & dickinsonite & arrojadite \\
\hline $1^{\text {st }}$ suffix & $\mathrm{Ba}$ & $\mathrm{Ba}$ & $\mathrm{Ba}$ & $\mathrm{Ba}$ & $\mathrm{Ba}$ & $\mathrm{Ba}$ & $\mathrm{Ba}$ & $\mathrm{Ba}$ \\
\hline $2^{\text {nd }}$ suffix & $\mathrm{Na}$ & $\mathrm{Na}$ & $\mathrm{Na}$ & $\mathrm{Na}$ & $\mathrm{Na}$ & $\mathrm{Na}$ & $\mathrm{Na}$ & $\mathrm{Na}$ \\
\hline $\mathrm{P}_{2} \mathrm{O}_{5 \text { EMPA-WDS }}$ & 40.31 & 40.45 & 40.36 & 40.14 & 40.13 & 40.01 & 39.84 & 40.39 \\
\hline $\mathrm{P}_{2} \mathrm{O}_{5 \text { calc. }}$ & 38.82 & 38.98 & 39.10 & 38.76 & 39.03 & 38.71 & 38.36 & 38.88 \\
\hline $\mathrm{TiO}_{2}$ & 0.05 & 0.06 & 0.05 & 0.06 & 0.06 & 0.09 & 0.04 & 0.06 \\
\hline $\mathrm{Al}_{2} \mathrm{O}_{3}$ & 2.28 & 2.39 & 2.44 & 2.38 & 2.44 & 2.40 & 2.41 & 2.39 \\
\hline $\mathrm{FeO}$ & 25.50 & 25.57 & 25.89 & 22.52 & 21.56 & 20.86 & 19.98 & 23.41 \\
\hline $\mathrm{MnO}$ & 16.46 & 16.59 & 16.47 & 19.52 & 20.48 & 20.82 & 20.91 & 18.41 \\
\hline $\mathrm{ZnO}$ & 0.29 & 0.19 & 0.20 & 0.11 & 0.21 & 0.13 & 0.24 & 0.19 \\
\hline $\mathrm{MgO}$ & 0.11 & 0.10 & 0.12 & 0.15 & 0.13 & 0.11 & 0.12 & 0.13 \\
\hline $\mathrm{CaO}$ & 2.06 & 2.04 & 2.03 & 1.92 & 1.91 & 1.92 & 2.08 & 2.07 \\
\hline $\mathrm{SrO}$ & 1.69 & 1.71 & 1.70 & 0.59 & 0.47 & 0.56 & 0.18 & 1.06 \\
\hline $\mathrm{BaO}$ & 3.17 & 3.03 & 3.11 & 4.96 & 5.39 & 5.30 & 6.32 & 4.33 \\
\hline $\mathrm{Li}_{2} \mathrm{O}$ LA-ICP-MS & 0.28 & 0.28 & 0.28 & 0.28 & 0.28 & 0.28 & 0.28 & 0.28 \\
\hline $\mathrm{Na}_{2} \mathrm{O}$ & 5.48 & 5.48 & 5.37 & 5.36 & 5.41 & 5.44 & 5.36 & 5.46 \\
\hline $\mathrm{K}_{2} \mathrm{O}$ & 0.96 & 0.94 & 0.89 & 0.82 & 0.78 & 0.81 & 0.63 & 0.84 \\
\hline $\mathrm{H}_{2} \mathrm{O}_{\text {calc. }}$ & 0.62 & 0.59 & 0.63 & 0.59 & 0.63 & 0.61 & 0.57 & 0.60 \\
\hline $\mathrm{F}$ & 1.30 & 1.36 & 1.29 & 1.34 & 1.29 & 1.30 & 1.36 & 1.33 \\
\hline $\mathrm{O}=\mathrm{F}$ & -0.55 & -0.57 & -0.54 & -0.56 & -0.54 & -0.55 & -0.57 & -0.56 \\
\hline Total & 98.52 & 98.74 & 99.03 & 98.80 & 99.62 & 98.79 & 98.27 & 98.88 \\
\hline $\mathrm{P}^{5+}$ & 12.000 & 12.000 & 12.000 & 12.000 & 12.000 & 12.000 & 12.000 & 12.000 \\
\hline $\mathrm{Ti}^{4+}$ & 0.981 & 1.024 & 1.042 & 1.026 & 1.044 & 1.036 & 1.050 & 1.027 \\
\hline $\mathrm{Al}^{3+}$ & 0.014 & 0.016 & 0.014 & 0.016 & 0.016 & 0.025 & 0.011 & 0.016 \\
\hline Sum $A l$ & 0.995 & 1.040 & 1.056 & 1.042 & 1.060 & 1.061 & 1.061 & 1.043 \\
\hline $\mathrm{Fe}\left(\mathrm{Fe}^{2+}\right)$ & 7.787 & 7.776 & 7.849 & 6.887 & 6.575 & 6.388 & 6.174 & 7.138 \\
\hline $\mathrm{Mn}^{2+}$ & 5.091 & 5.110 & 5.057 & 6.046 & 6.300 & 6.457 & 6.544 & 5.685 \\
\hline $\mathrm{Zn}^{2+}$ & 0.078 & 0.051 & 0.054 & 0.030 & 0.056 & 0.035 & 0.065 & 0.051 \\
\hline $\mathrm{Mg}^{2+}$ & 0.060 & 0.054 & 0.065 & 0.082 & 0.070 & 0.060 & 0.066 & 0.071 \\
\hline $\mathrm{Li}^{+}$ & 0.411 & 0.409 & 0.408 & 0.412 & 0.409 & 0.412 & 0.416 & 0.411 \\
\hline Sum $M$ & 13.427 & 13.400 & 13.433 & 13.457 & 13.410 & 13.352 & 13.265 & 13.356 \\
\hline $\mathrm{Ca}^{2+}$ & 0.806 & 0.795 & 0.788 & 0.752 & 0.743 & 0.753 & 0.823 & 0.809 \\
\hline $\mathrm{Sr}^{2+}$ & 0.358 & 0.361 & 0.357 & 0.125 & 0.099 & 0.119 & 0.039 & 0.224 \\
\hline $\mathrm{Ba}^{2+}$ & 0.454 & 0.432 & 0.442 & 0.711 & 0.767 & 0.760 & 0.915 & 0.619 \\
\hline $\mathrm{Na}^{+}$ & 3.879 & 3.863 & 3.774 & 3.800 & 3.809 & 3.862 & 3.840 & 3.859 \\
\hline $\mathrm{K}^{+}$ & 0.447 & 0.436 & 0.412 & 0.383 & 0.361 & 0.378 & 0.297 & 0.391 \\
\hline Sum $X$ & 5.944 & 5.887 & 5.773 & 5.771 & 5.779 & 5.872 & 5.914 & 5.902 \\
\hline Sum $M+X$ & 19.371 & 19.287 & 19.206 & 19.228 & 19.189 & 19.224 & 19.179 & 19.258 \\
\hline Li calc. & 0.406 & 0.543 & 0.590 & 0.551 & 0.610 & 0.616 & 0.611 & 0.556 \\
\hline${ }^{\mathrm{M}} \mathrm{Fe}^{*}$ & 0.427 & 0.400 & 0.433 & 0.457 & 0.410 & 0.352 & 0.265 & 0.356 \\
\hline Sum $X^{2+}$ & 1.618 & 1.588 & 1.587 & 1.588 & 1.609 & 1.632 & 1.777 & 1.652 \\
\hline Sum cat. & 32.366 & 32.327 & 32.262 & 32.269 & 32.249 & 32.284 & 32.240 & 32.301 \\
\hline $\mathrm{OH}^{-}$ & 1.499 & 1.436 & 1.521 & 1.450 & 1.518 & 1.495 & 1.411 & 1.466 \\
\hline $\mathrm{F}^{-}$ & 1.501 & 1.564 & 1.479 & 1.550 & 1.482 & 1.505 & 1.589 & 1.534 \\
\hline $\mathrm{O}\left(\mathrm{O}^{2-}\right)$ & 47.000 & 47.000 & 47.000 & 47.000 & 47.000 & 47.000 & 47.000 & 47.000 \\
\hline Sum of anions & 50.000 & 50.000 & 50.000 & 50.000 & 50.000 & 50.000 & 50.000 & 50.000 \\
\hline $\mathrm{F} /(\mathrm{F}+\mathrm{OH}) W$ & 0.751 & 0.782 & 0.740 & 0.775 & 0.741 & 0.753 & 0.795 & 0.767 \\
\hline $\mathrm{Mn} /(\mathrm{Mn}+\mathrm{Fe})$ & 0.395 & 0.397 & 0.392 & 0.467 & 0.489 & 0.503 & 0.515 & 0.443 \\
\hline
\end{tabular}

$X=A 1+A 2+B 1+B 2+C a+N a$ cation sites; formulae based on $\mathrm{P}=12$ apfu and $(\mathrm{OH}+\mathrm{F})=3$ apfu

$\mathrm{S}, \mathrm{As}, \mathrm{Si}$, and $\mathrm{Cl}$ contents are below detection limit

Mn (10 to 11 wt. \% MnO, 0.34-0.38 apfu $\mathrm{Mn}$ ) which shows a positive correlation with Fe concentrations (1.8 to 2.6 wt. \% $\mathrm{FeO}, 0.06-0.09$ apfu $\mathrm{Fe}$ ), possibly due to $(\mathrm{Fe}, \mathrm{Mn}, \mathrm{Mg}) \mathrm{Ca}_{-1}$ substitution (Fig. $6 \mathrm{~b}-\mathrm{c}$ ). 

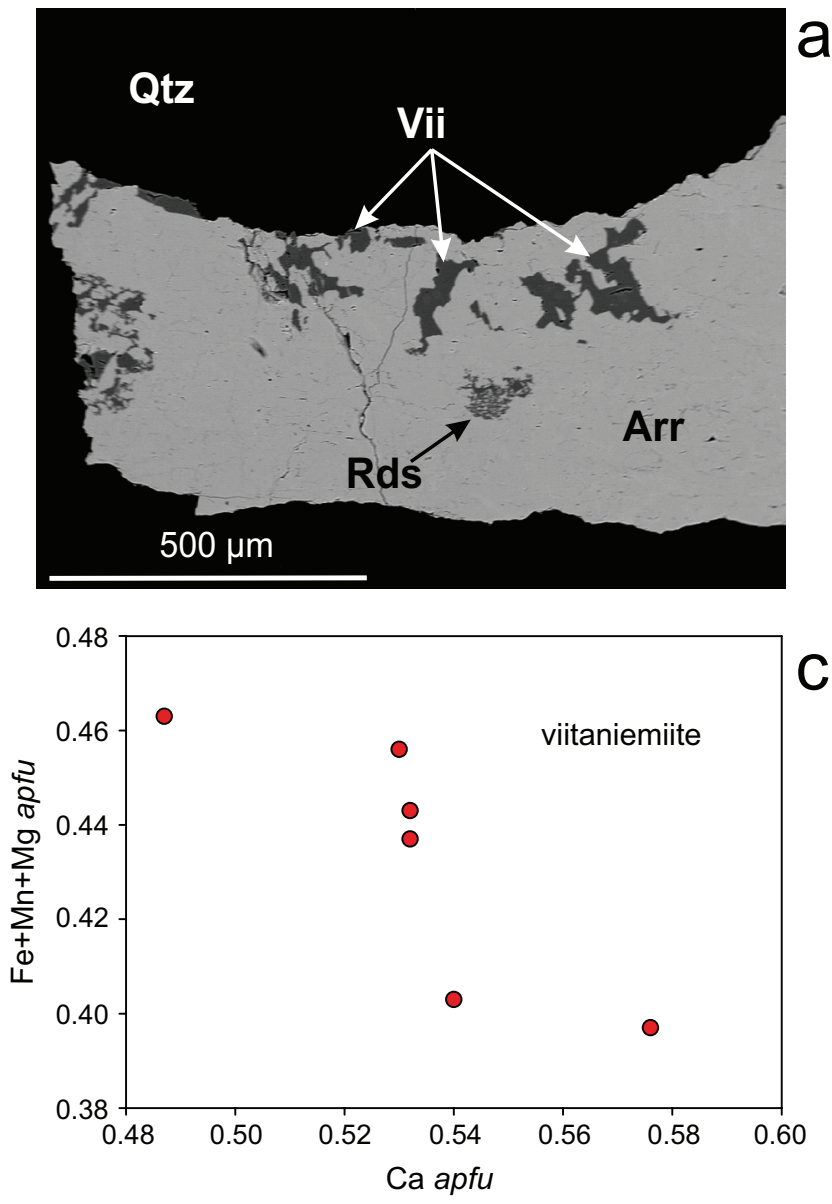

\section{Discussion and conclusions}

The studied association represents $\mathrm{F}$-rich, $\mathrm{Mn}, \mathrm{Fe}, \mathrm{Ca}-$ phosphate minerals which precipitated with massive quartz and minor Fe-rich rhodochrosite, albite, muscovite, fluorite and sulphide minerals. Fluorapatite is probably the oldest, as it is partly replaced by triplite (Fig. 2a-b). The mutual textural relationships between other phases are not unambiguous but indicate near contemporary origin of all the remaining phosphates together with quartz, rhodochrosite and other associated hydrothermal minerals. Such mineral assemblage as well as textural relationships indicates a relatively high-temperature, early hydrothermal (subsolidus) origin. Similar post-magmatic, early hydrothermal origin of triplite in granite-related quartz veins was proposed, for example, in Tigrinoe tin deposit, Russia (Gonevchuk et al. 2005), Huber stock near Horní Slavkov, Czech Republic (Sejkora et al. 2006), or Panasqueira, Portugal (Isaacs and Peacor 1981; Milá and Fabre 2014).

Both described members of arrojadite group ["fluorarrojadite-(BaNa)" and "fluordickinsonite-(BaNa)"] are still not officially approved as valid minerals by CNMNC IMA. However, "fluorarrojadite-(BaNa)" composition is documented from the Sidi-bou-Kricha pegmatite,

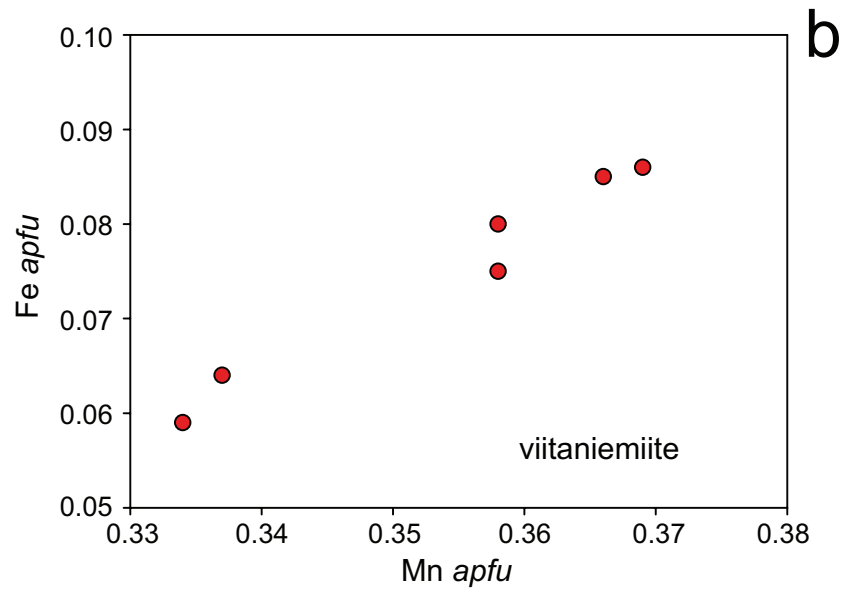

Fig. 6 Viitaniemiite from Gemerská Poloma. a - Irregular aggregates of viitaniemiite (Vii) and minor rhodochrosite (Rds) enclosed in minerals of the arrojadite group (Arr), BSE image. Substitution diagrams: $\mathbf{b}-\mathrm{Mn}$ vs. $\mathrm{Fe} ; \mathbf{c}-\mathrm{Ca}$ vs. $\mathrm{Fe}+\mathrm{Mn}+\mathrm{Mg}$.

Morocco and it is has been identified as a potential new mineral species (Chopin et al. 2006).

Unapproved fluor-dominant members of the arrojadite group ["fluorarrojadite-(BaNa)" and "fluorarrojadite(SrNa)"] were also described from topaz-zinnwaldite microgranite of the Gemeric type from Surovec (Petrík et al. 2011), only $\sim 8 \mathrm{~km} \mathrm{NNE}$ of the locality studied here. "Fluordickinsonite-(BaNa)" compositions were also noted from the Surovec microgranite (Petrík et al. 2011) but the published crystallochemical formulae of the two analyses showed $\mathrm{Fe}>\mathrm{Mn}$ and $\mathrm{Mn} /(\mathrm{Mn}+\mathrm{Fe})<$ $0.5(0.48)$ in the $M$ site; therefore they could be classified as "fluorarrojadite-(BaNa)" and "fluorarrojadite-( $\mathrm{SrNa})$ ".

Viitaniemiite represents a rare $\mathrm{Na}-\mathrm{Ca}-\mathrm{Al}$ phosphate mineral, firstly described from granitic pegmatites in Eräjärvi area, Finnland (Lahti 1981). Our compositions of viitaniemiite show a distinct Mn enrichment (10 to 11 wt. \% $\mathrm{MnO}$ ) which is comparable with viitaniemiite from the Eräjärvi pegmatites (10-12 wt. \% MnO; Lahti $1981)$ and from the topaz-zinnwaldite microgranite in Surovec (8-10 wt. \% MnO; Petrík et al. 2011). On the other hand, viitaniemiite from alkali silicocarbonatites in Francon quarry, Montréal, Canada does not contain any detectable Mn (Ramik et al. 1983). 
Phosphate minerals in quartz veins from the Gemerská Poloma, Slovakia

Experimental results indicate a relatively high solubility of P-rich and F-bearing magmatic liquids in aqueous fluids and subsequent precipitation of fluorite, topaz, apatite, and triplite in greisens, hydrothermal veins and miarolitic cavities associated with the P-rich granites (Webster et al. 1998). The $\mathrm{Fe}-\mathrm{Mn}$ phosphate minerals (childrenite-eosphorite, zwiesselite and triphylite) were formed also during latemagmatic crystallization of the strongly P- and F-enriched residual melt in extremely fractionated, P-rich granite at Podlesí, Czech Republic (Breiter et al. 1997, 2005). Moreover, primary magmatic P-rich alkali feldspars (especially orthoclase) could be an alternative source of phosphorus (London 1992; Frýda and Breiter 1995), released during their post-magmatic alteration by F-rich fluids. This process would enable crystallization of early hydrothermal Fe-rich phosphates (Breiter et al. 1997) or fluorapatite (Broska et al. 2004).

Consequently, in the case of Gemerská Poloma we suggest a precipitation of the phosphates and associated minerals from alkali-, P- and F-rich post-magmatic to early hydrothermal, relatively high-temperature fluids which altered primary magmatic minerals (especially Li-rich micas and alkali feldspars) and liberated some elements ( $\mathrm{Fe}, \mathrm{Mn}, \mathrm{Al}, \mathrm{Ca}, \mathrm{Ba}, \mathrm{Sr}, \mathrm{Na}$ and $\mathrm{K}$ ) from the adjacent granite.

Acknowledgements. We would like to acknowledge Radek Škoda (Masaryk University, Brno), Peter Bačík (Comenius University, Bratislava) and Pavel Škácha (Mining Museum, Přibram) for their support of this study. Both referees, Fernando Cámara and Igor Petrík, same as handling editor Igor Broska and the editor-in-chief Vojtěch Janoušek, are highly acknowledged for comments and suggestions that helped to improve the manuscript. This work was financially supported by the Ministry of Culture of the Czech Republic (DKRVO 2015/02; National Museum 00023272).
Tab. 7 Chemical composition of viitaniemiite from Gemerská Poloma (in wt. \%)

\begin{tabular}{|c|c|c|c|c|c|c|}
\hline Anal. \# & 22 & 24 & 25 & 26 & 27 & 28 \\
\hline $\mathrm{P}_{2} \mathrm{O}_{5}$ & 29.01 & 29.35 & 30.00 & 29.99 & 29.64 & 29.37 \\
\hline $\mathrm{Al}_{2} \mathrm{O}_{3}$ & 20.74 & 20.57 & 20.54 & 20.37 & 20.58 & 20.76 \\
\hline $\mathrm{FeO}$ & 2.50 & 2.24 & 1.94 & 2.62 & 1.78 & 2.37 \\
\hline $\mathrm{MnO}$ & 10.61 & 10.49 & 10.11 & 11.06 & 9.89 & 10.52 \\
\hline $\mathrm{MgO}$ & 0.09 & 0.07 & 0.04 & 0.13 & 0.06 & 0.09 \\
\hline $\mathrm{CaO}$ & 12.16 & 12.33 & 12.80 & 11.53 & 13.48 & 12.35 \\
\hline $\mathrm{Na}_{2} \mathrm{O}$ & 12.67 & 12.74 & 12.70 & 12.15 & 12.26 & 12.80 \\
\hline $\mathrm{H}_{2} \mathrm{O}^{*}$ & 3.46 & 3.60 & 3.78 & 3.61 & 3.60 & 3.49 \\
\hline $\mathrm{F}^{2}$ & 16.00 & 15.97 & 16.12 & 16.46 & 16.20 & 16.22 \\
\hline $\mathrm{O}=\mathrm{F}$ & -6.74 & -6.72 & -6.79 & -6.93 & -6.82 & -6.83 \\
\hline Total & 100.50 & 100.64 & 101.24 & 100.99 & 100.67 & 101.14 \\
\hline $\mathrm{P}^{5+}$ & 1.000 & 1.000 & 1.000 & 1.000 & 1.000 & 1.000 \\
\hline $\mathrm{Al}^{3+}$ & 0.995 & 0.976 & 0.953 & 0.946 & 0.967 & 0.984 \\
\hline $\mathrm{Fe}^{2+}$ & 0.085 & 0.075 & 0.064 & 0.086 & 0.059 & 0.080 \\
\hline $\mathrm{Mn}^{2+}$ & 0.366 & 0.358 & 0.337 & 0.369 & 0.334 & 0.358 \\
\hline $\mathrm{Mg}^{2+}$ & 0.005 & 0.004 & 0.002 & 0.008 & 0.004 & 0.005 \\
\hline $\mathrm{Ca}^{2+}$ & 0.530 & 0.532 & 0.540 & 0.487 & 0.576 & 0.532 \\
\hline Sum $M$ & 0.986 & 0.969 & 0.943 & 0.950 & 0.973 & 0.975 \\
\hline $\mathrm{Na}^{+}$ & 1.000 & 0.994 & 0.970 & 0.928 & 0.947 & 0.998 \\
\hline $\mathrm{OH}^{-}$ & 0.940 & 0.967 & 0.993 & 0.950 & 0.958 & 0.937 \\
\hline $\mathrm{F}^{-}$ & 2.060 & 2.033 & 2.007 & 2.050 & 2.042 & 2.063 \\
\hline $\operatorname{Sum} X$ & 3.000 & 3.000 & 3.000 & 3.000 & 3.000 & 3.000 \\
\hline $\mathrm{O}^{2-}$ & 3.980 & 3.930 & 3.857 & 3.831 & 3.896 & 3.951 \\
\hline Sum cat. & 3.981 & 3.939 & 3.866 & 3.824 & 3.887 & 3.957 \\
\hline Sum an. & 6.980 & 6.930 & 6.857 & 6.831 & 6.896 & 6.951 \\
\hline $\mathrm{Mn} /(\mathrm{Mn}+\mathrm{Ca})$ & 0.41 & 0.40 & 0.38 & 0.43 & 0.37 & 0.40 \\
\hline
\end{tabular}

Formulae based on $\mathrm{P}=1$ apfu and $(\mathrm{OH}+\mathrm{F})=3$ apfu

$\mathrm{S}, \mathrm{As}, \mathrm{Si}, \mathrm{Ti}, \mathrm{Zn}, \mathrm{Sr}, \mathrm{Ba}, \mathrm{K}, \mathrm{Cl}$ were below detection limit

\section{References}

Baijot M, Hatert F, Philippo S (2012) Mineralogy and geochemistry of phosphates and silicates in the Sapucaia pegmatite, Minas Gerais, Brazil: genetic implications. Canad Mineral 50: 1531-1554

Bajaník Š, Ivanička J, Mello J, Pristaš J, Reichwalder P, SNOPKo L, VozÁr J, VozÁrová A (1984) Geological map of the Slovenské Rudohorie Mts. - eastern part 1:50 000. Dionýz Štúr Institute of Geology, Bratislava

Breiter K, Frýda J, Seltmann R, Thomas R (1997) Mineralogical evidence for two magmatic stages in the evolution of an extremely fractionated P-rich rare-metal granite: the Podlesí stock, Krušné Hory, Czech Republic. J Petrol 38: 1723-1739

Breiter K, Müller A, Leichmann J, Gabašová A (2005) Textural and chemical evolution of a fractionated granitic system: the Podlesí stock, Czech Republic. Lithos 80: $323-345$

Breiter K, Broska I, Uher P (2015) Intensive low-temperature tectono-hydrothermal overprint of peraluminous rare-metal granite: a case study from the Dlhá dolina Valley (Gemericum, Slovakia). Geol Carpath 66: 19-36 
Broska I, Williams CT, Uher P, Konečný P, Leichmann J (2004) The geochemistry of phosphorus in different granite suites of the Western Carpathians, Slovakia: the role of apatite and P-bearing feldspar. Chem Geol 205: 1-15

Cámara F, Oberti R, Chopin C, Medenbach O (2006) The arrojadite enigma: I. A new formula and a new model for the arrojadite structure. Amer Miner 91: 1249-1259

Chopin C, Oberti R, Cámara F (2006) The arrojadite enigma: II. Compositional space, new members, and nomenclature of the group. Amer Miner 91: 1260-1270

Della Ventura G, Bellatreccia F, Radica F, Chopin C, OBERTI R (2014) The arrojadite enigma III. The incorporation of volatiles: a polarised FTIR spectroscopy study. Eur J Mineral 26: 679-688

DianišKa I, Breiter K, Broska I, Kubiš M, Malachovský P (2002) First phosphorous-rich $\mathrm{Nb}-\mathrm{Ta}-\mathrm{Sn}$-specialised granite from the Carpathians - Dlhá dolina Valley granite pluton, Gemeric Superunit. Geol Carpath 53: Special Issue (CD-ROM).

DianišKa I, Uher P, Hurai V, Huraiová M, Frank W, KoneČNÝ P, KRÁL J (2007) Mineralization of rare-metal granites. In: HuraI V (ed) Sources of Fluids and Origin of Mineralizations in the Gemeric Unit. Open file report, Dionýz Štúr Institute of Geology, Bratislava, pp 254-330 (in Slovak)

Downs RT (2006) The RRUFF Project: an integrated study of the chemistry, crystallography, Raman and infrared spectroscopy of minerals. Program and Abstracts of the $19^{\text {th }}$ General Meeting of the International Mineralogical Association in Kobe, Japan, O03-13

FrÝDA J, BREITER K (1995) Alkali feldspars as a main phosphorus reservoirs in rare-metal granites: three examples from the Bohemian Massif (Czech Republic). Terra Nova 7: 315-320

GaAb AS, Todt W, Poller U (2006) CLEO: Common lead evaluation using Octave. Comput Geosci 32: 993-1003

Gonevchuk VG, Korostelev PG, Semenyak BI (2005) Genesis of the Tigrinoe tin deposit (Russia). Geol Ore Deposits 47: 223-237

Isaacs AM, Peacor DR (1981) Panasqueiraite, a new mineral: The $\mathrm{OH}$-equivalent of isokite. Canad Mineral 19: 389-392

KıLíK J (1997) Geological characteristic of the talc deposit in Gemerská Poloma-Dlhá dolina. Acta Montan Slovaca 2: 71-80 (in Slovak)

КонÚt M, Stein H (2005) Re-Os molybdenite dating of granite-related Sn-W-Mo mineralisation at Hnilec, Gemeric Superunit, Slovakia. Miner Petrol 85: 117-129

Kubiš M, BRoska I (2005) The role of boron and fluorine in evolved granitic rock systems (on the example of the Hnilec area, Western Carpathians). Geol Carpath 56: 193-204

Kubiš M, Broska I (2010) The granite system near Betliar village (Gemeric Superunit, Western Carpathians): evolution of a composite silicic reservoir. J Geosci 55: 131-148

LAHTI SI (1981) On the granitic pegmatites of the Eräjärvi area in Orivesi, southern Finland. Geol Surv Finland Bull 314: 1-82

Laugier J, Bochu B (2011) LMGP - Suite of Programs for the Interpretation of X-ray Experiments. Accessed on December 2, 2015, at http://www.ccp14.ac.uk/tutorial//mgp

Lazic B, Armbruster T, Chopin C, Grew ES, Baronnet A, Palatinus L (2014) Superspace description of wagneritegroup minerals $(\mathrm{Mg}, \mathrm{Fe}, \mathrm{Mn})_{2}\left(\mathrm{PO}_{4}\right)(\mathrm{F}, \mathrm{OH})$. Acta Cryst B70: 243-258

LLOREns T, Moro MC (2012) Fe-Mn phosphate associations as indicators of the magmatic-hydrothermal and supergene evolution of the Jálama Batholith in the Navasfrías Sn-W District, Salamanca, Spain. Mineral Mag 76: 1-24

London D (1992) Phosphorus in S-type magmas: the $\mathrm{P}_{2} \mathrm{O}_{5}$ content of feldspars from peraluminous granites, pegmatites and rhyolites. Amer Miner 77: 126-145

LONDON D (1997) Estimating abundances of volatile and other mobile components in evolved silicic melts through mineral-melt equilibria. J Petrol 38: 1691-1706

Milá CC, Fabre J (2014) The Panasqueira mines, Castelo Branco district, Portugal. Mineral Rec 45: 11-55

Petrasová K, Faryad SW, Jeřábek P, ŽáčKová E (2007) Origin and metamorphic evolution of magnesite-talc and adjacent rocks near Gemerská Poloma, Slovak Republic. J Geosci 52: 125-132

Petrík I, Kohút M (1997) The evolution of granitoid magmatism during the Hercynian orogen in the Western Carpathians. In: Grecula P, Hovorka D, Putiš M (eds) Geological Evolution of the Western Carpathians. Miner Slov, Monogr, 235-252

Petrík I, Kubiš M, KoneČnÝ P, Broska I, MalachovskÝ P (2011) Rare phosphates from the Surovec topaz-Li mica microgranite, Gemeric Unit, Western Carpathians, Slovak Republic: role of $\mathrm{F} / \mathrm{H}_{2} \mathrm{O}$ of the melt. Canad Mineral 49: $521-540$

Petrík I, Č́́k Š, Miglierini M, Vaculovič T, DianišKa I, OzDín D (2014) Alpine oxidation of lithium micas in Permian S-type granites (Gemeric unit, Western Carpathians, Slovakia). Mineral Mag 78: 507-533

Piccoli PM, CANDELA PA (2002) Apatite in igneous systems. In: Kohn MJ, Rakovan J, Hughes JM (eds) Phosphates: Geochemical, Geobiological, and Materials Importance. Mineralogical Society of America, Reviews in Mineralogy \& Geochemistry 48, Washington, pp 256-292

Pouchou JL, PIchoIR F (1985) "PAP" ( $\varphi \rho Z)$ procedure for improved quantitative microanalysis. In: ARMSTRONG JT (ed) Microbeam Analysis. San Francisco Press, pp 104-106

Poller U, Uher P, Broska I, Plašienka D, Janák M (2002) First Permian-Early Triassic zircon ages for tin-bearing granites from the Gemeric Unit (Western Carpathians, 
Slovakia): connection to the post-collisional extension of the Variscan orogen and S-type granite magmatism. Terra Nova 14: 41-48

Ramik RA, Sturman BD, Roberts AC, Dunn PJ (1983) Viitaniemiite from the Francon quarry, Montreal, Quebec. Can Mineral 21: 499-502

Roda-Robles E, Pesquera A, Gil-Crespo P, Torres-Ruiz J (2012) The Puentemocha beryl-phosphate granitic pegmatite, Salamanca, Spain: internal structure, petrography and mineralogy. Canad Mineral 50: 1573-1597

SejKora J, ŠKoda R, Ondruš P, Beran P, Süsser C (2006) Mineralogy of phosphate accumulations in the Huber stock, Krásno ore district, Slavkovský les area, Czech Republic. J Czech Geol Soc 51: 103-147

Uher P, Broska I (1996) Post-orogenic Permian granitic rocks in the Western Carpathian-Pannonian area: geochemistry, mineralogy and evolution. Geol Carpath 47: 311-321

WALDROP L (1969) The crystal structure of triplite, $(\mathrm{Mn}, \mathrm{Fe})_{2} \mathrm{FPO}_{4}$. Z Kristallogr 130: 1-14

Webster JD, Thomas R, Veksler I, Rhede D, Seltmann R, Förster H-J (1998) Late-stage processes in P- or F-rich granitic magmas. Acta Univ Carol, Geol 42: $181-188$ 